\title{
1 Start codon context controls translation initiation in the fungal kingdom
}

2 Edward Wallace ${ }^{2 *}$, Corinne Maufrais ${ }^{1,3}$, Jade Sales-Lee ${ }^{4}$, Laura Tuck ${ }^{2}$, Luciana de Oliveira ${ }^{1}$,

3 Frank Feuerbach $^{6}$, Frédérique Moyrand ${ }^{1}$, Prashanthi Natarajan ${ }^{4}$, Hiten D. Madhani ${ }^{4,5^{*}}$,

4 Guilhem Janbon ${ }^{1 *}$

7 1. Institut Pasteur, Unité Biologie des ARN des Pathogènes Fongiques, Département de

8 Mycologie, F-75015, Paris, France

9 2. Institute for Cell Biology and SynthSys, School of Biological Sciences, University of

10 Edinburgh, UK

11 3. Institut Pasteur, HUB Bioinformatique et Biostatistique, C3BI, USR 3756 IP CNRS, F-75015,

12 Paris, France

13 4. Department of Biochemistry and Biophysics, University of California at San Francisco, San

14 Francisco, California 94158, USA

15 5. Chan-Zuckerberg Biohub, San Francisco, CA 94158, USA

166 Institut Pasteur, Unité Génétique des Interactions Macromoléculaire, Département

17 Génome et Génétique, F-75015, Paris, France

$18 *$ Corresponding authors

19 ? these authors contribute equality to this work and should be considered as co-first authors 


\section{Abstract}

2 Eukaryotic protein synthesis initiates at a start codon defined by an AUG and its surrounding

3 Kozak sequence context, but studies of S. cerevisiae suggest this context is of little

4 importance in fungi. We tested this concept in two pathogenic Cryptococcus species by

5 genome-wide mapping of translation and of mRNA 5' and 3' ends. We observed that

6 upstream open reading frames (uORFs) are a major contributor to translation repression,

7 that uORF use depends on the Kozak sequence context of its start codon, and that uORFs

8 with strong contexts promote nonsense-mediated mRNA decay. Numerous Cryptococcus

9 mRNAs encode predicted dual-localized proteins, including many aminoacyl-tRNA

10 synthetases, in which a leaky AUG start codon is followed by a strong Kozak context in-frame

11 AUG, separated by mitochondrial-targeting sequence. Further analysis shows that such dual-

12 localization is also predicted to be common in Neurospora crassa. Kozak-controlled

13 regulation is correlated with insertions in translational initiation factors in fidelity-

14 determining regions that contact the initiator tRNA. Thus, start codon context is a signal that

15 programs the expression and structures of proteins in fungi. 


\section{Introduction}

2 Fungi are important in the fields of ecology, medicine, and biotechnology. With 4 million

3 predicted fungal species, this kingdom is the most diverse of the domain Eukarya. Recent

4 initiatives such as the 1000 Fungal Genomes Project at the Joint Genome Institute, or the

5 Global Catalogue of Microorganisms, which aims to produce 2500 complete fungal genomes

6 in the next 5 years, will result in a deluge of genome sequence data $(1,2)$. Comparative

7 analysis of coding sequences enables the generation of hypotheses on genome biology and

8 evolution (3-6). However, these analyses intrinsically depend on the quality of the coding

9 gene identification and annotation, which have limitations. First, they depend on automatic

10 sequence comparisons, which limit the identification of clade-specific genes. Second, fungal

11 genes generally contain introns whose positions are difficult to predict based on the genome

sequence alone (7). An uncertain intron annotation results in a poor annotation of the

coding region extremities, which are generally less evolutionary conserved (8). Third,

annotation pipelines only predict plausible open reading frames (ORFs), initially for yeast a

contiguous stretch of at least 100 codons starting with an AUG codon and ending with a stop

codon (9). These approaches do not reveal which ORFs are translated to protein, and are

biased against short ORFs (10).

The common assumption is that the first AUG of a fungal ORF is used as the translation start

codon, yet non-AUG start codons have been observed in every studied eukaryote, including

the fungi S. cerevisiae, C. albicans, S. pombe, and N. crassa (11-16). In metazoans the rules

for selection of AUG start codons were discovered by Kozak: AUGs are efficiently selected by 
1 The influence of the motif on the efficiency of translation is organism-dependent. Although

2 in metazoan cells the Kozak context has a major effect on translation initiation (19), in S.

3 cerevisiae translation usually starts at the first AUG in the mRNA sequence, the strong

4 aaAAUG sequence context only weakly affects endogenous protein output (20), and AUG

5 sequence context somewhat modulates protein output from reporter mRNAs $(21,22)$. In

6 fact, translation start codon context has a larger effect on non-AUG start codon usage (23).

7 These "Saccharomyces rules" have been considered as the paradigm for fungi, but relevant

8 data are lacking in other species of fungi.

9 Weak or inefficient start codons near the 5'end of mRNA can give rise to translational regulation, explained by the scanning model of eukaryotic translation initiation. Translation starts by the pre-initiation complex binding mRNA at the $5^{\prime}$ cap and then scanning the transcript leader (TL) sequence until it identifies a start codon, at which translation initiates (19). Here we call the $5^{\prime}$ regulatory region of mRNA the TL rather than the $5^{\prime}$ UTR, because short "upstream" ORFs in this region can be translated (24). The pre-initiation complex sediments at 43S, and comprises the small ribosomal subunit, methionyl initiator tRNA, and numerous eukaryotic translation initiation factors (elFs). Biochemical, genetic, and structural data indicate that elF1 and elF1A associate with the $43 S$ pre-initiation complex $(25,26)$. Recognition of the start codon involves direct interactions of elF1 and elF1A with the start codon context and initiator tRNA within a larger elF2/3/5-containing 48S pre-initiation complex. Start codon selection occurs when elF1 is replaced by elF5's N-terminus (27), then elF2 is released, the large ribosomal subunit joins catalyzed by elF5B, and translation begins (26). This work has been largely driven by studies in S. cerevisiae and metazoans. Although the core protein and RNA machinery of eukaryotic translation initiation is highly conserved, 
1 it is not understood how fungi quantitatively vary in the sequence, structure, and function of

2 their translation initiation machinery.

3 Cryptococcus are basidiomycete yeasts with a high density of introns in their coding genes

4 (28). These introns influence gene expression and genome stability (29-31). The current

5 genome annotation of pathogenic $C$. neoformans and deneoformans reference strains are

6 based on both automatic and manual curations of gene structures using RNA-Seq data (32,

7 33). Although the high degree of interspecies conservation of intron numbers and positions

8 within coding sequences suggest that these annotations are reliable (33), the regulatory

9 regions (transcript leader and 3' UTRs) at transcript extremities are less well identified. In

10 fact, most fungal genomes lack complete transcript annotations, and thus we do not know

11 how regulatory structure varies across fungi.

12 In this paper, we experimentally determine the beginning and the end of both coding

13 regions and of transcripts in two Cryptococcus species, providing an important genomic

14 resource for the field. Furthermore, our joint analysis of TL sequences and translation

15 identifies a Kozak sequence context that regulates start codon selection, affecting upstream

16 ORF regulation and also alternative protein targeting to mitochondria. Comparison with

17 other fungal genomes revealed that these types of regulation are common in this kingdom:

the first AUG of an mRNA or an ORF is not always the major start codon in fungi. These

19 studies demonstrate that, in contrast to the situation in S. cerevisiae, start codon sequence

20 context is an important gene regulatory signal that programs the levels and structures of

21 proteins in the fungal kingdom. 
2 RESULTS

\section{Delineation of transcript ends in C. neoformans and C. deneoformans}

4 To annotate the extremities of the coding genes in $C$. neoformans and $C$. deneoformans, we

5 mapped the 5' ends (Transcription Start Sites; TSS) with TSS-Seq (34), the 3' ends

6 (Polyadenylation sites; PAS) with QuantSeq 3'mRNA-Seq, and sequenced the same samples

7 with stranded mRNA-Seq. These experiments were done in biological triplicate from cells

8 growing at two temperatures $\left(30^{\circ} \mathrm{C}\right.$ and $\left.37^{\circ} \mathrm{C}\right)$ and two stages of growth (exponential and

9 early stationary phases) with external normalization with spike-in controls.

10 We identified $4.7 \times 10^{6}$ unique TSSs and $6.3 \times 10^{4}$ unique PASs in C. neoformans. Clustering of

11 these positions revealed between 27,339 and 42,720 TSS clusters and between 9,217 and 16,697 PAS clusters depending on the growth conditions (Table S1). We used the clusters associated with the coding genes to produce an initial annotation, using the most distal TSS and PAS clusters for each gene. The predicted positions which changed the extremities of the genes by more than 100 bp were manually curated $(n=1131$ and $n=286$ for the TSS and PAS, respectively). We then selected the most prominent clusters that represented at least $10 \%$ of the normalized reads count per coding gene in at least one condition. Finally, the most distal of these TL-TSS and 3'UTR-PA clusters were labeled as the 5'and 3' ends of the coding genes for our final annotation (Table S1). For the genes for which no TL-TSS cluster or no 3'UTR-PAS cluster could be identified, we maintained the previous annotation. We used the same strategies for $C$. deneoformans and obtained similar results (Table S1). As expected, most of the TSS clusters (62\%) were associated with the TL whereas most of the PAS clusters (82\%) were associated with the 3'UTR of the coding genes (Table S1). We analyzed the 3'UTR sequences, confirming the ATGHAH motif associated with the PAS (32). 
1 In addition, as previously observed in other systems (35) a (C/T)(A/G)-rich motif was

2 associated with the maxima of these transcription start site clusters. Overall, $89 \%$ of the

3 coding genes have both their TL and 3'UTR sequences supported by identified TSS and PAS

4 clusters, respectively.

5 The analysis leads to a scheme of a stereotypical C. neoformans coding gene (Figure $1 \mathrm{~A}$ ). In

6 average, it is 2,305 bp long (median 2,008 bp) and contains 5.6 short introns (median 5) in its

7 sequence. As previously reported (28), these introns are short (63.4 nt in average) and

8 associated with conserved consensus motifs. The $C$. neoformans TL and 3'UTR have median

9 lengths of $107 \mathrm{nt}$ and $129 \mathrm{nt}$, respectively (180 nt and $189 \mathrm{nt}$, mean; Figure 1A,B). Only 887

10 and 429 genes contain one or more introns in their TL and 3'UTR sequence, respectively;

11 these introns are usually larger (118.3 nt) than those that interrupt the CDS. This gene structure is similar in $C$. deneoformans (Table S1) and there are good correlations between the 3'UTR and TL sizes of the orthologous genes in the two species (Figure 1C,D).

\section{More than a third of genes have upstream ORFs that affect translation}

The analysis of the TL sequences in C. neoformans revealed the presence of 10,286 AUG

triplets upstream (UAUG) of the annotated translation start codon (aAUG). We include

uAUGs that are either out-of-frame from the start codon, or in-frame but with an

intervening stop codon, which are very unlikely to encode a continuous polypeptide.

Strikingly, 2,942 genes possess at least one UAUG, representing $43 \%$ of the genes with an

annotated TL in C. neoformans (Figure 1F). A similar result was obtained in $C$. deneoformans, 
1 Translation initiation at UAUGs results in the translation of uORFs, which can regulate

2 expression of the main $\operatorname{ORF}(36,37)$. To evaluate the functionality of the UAUGs in

3 Cryptococcus, we generated riboprofiling data in both species and compared densities of

4 ribosome-protected fragments with those of sample-matched poly(A)+RNA. Our

5 riboprofiling data passes quality metrics of 3-nucleotide periodicity of reads on ORFs

6 indicating active translation by ribosomes, and appropriate read lengths of 26-30nt (Figure

7 S2.1).

8 Most genes have ribosome occupancy close to that predicted by their RNA abundance, and

9 restricted to the main $\mathrm{ORF}$, for example the most highly translated gene, translation

10 elongation factor eEF1 $\alpha / C N A G \_06125$ (Figure 2A,B). However, we observed dramatic examples of translation repression associated with uORFs in CNAG_06246, CNAG_03140 in

C. neoformans (Figure 2A,C,D). These patterns are conserved in their homologs in $C$.

deneoformans (Figure S2.2). Other spectacularly translationally repressed genes, contain conserved uORFs in addition to $5^{\prime}$ introns with alternative splicing or intronically expressed non-coding RNAs (Fig S2.3). In all these cases, high ribosomal occupancy on one or more UORFs is associated with low occupancy of the main ORF.

The uncharacterized gene CNAG_06246 has two AUG-encoded uORFs that are occupied by ribosomes, and a predicted C-terminal bZIP DNA-binding domain. This gene structure is reminiscent of the multi-uORF-regulated amino-acid responsive transcription factors CNAG_03140 has six uAUGs, with substantial ribosome occupancy only at the first. Interestingly, $N$. crassa has a sugar transporter in the same major facilitator superfamily 
1 regulated by a uORF (rco-3/sor-4, (39)), and sugar-responsive translational repression via

2 uORFs has been observed in plants (40).

3 Since these translationally repressed genes have multiple uAUGs, we investigated the

4 relationship between UAUGs and translation efficiency genome-wide. We observed a clear

5 negative relationship between the number of $\mathrm{UAUGs}$ and translation efficiency (Figure 2E,

6 S2.2E), suggesting an UAUG-associated negative regulation of gene expression in both

7 species.

8

9 Position relative to the TSS affects UAUG translation.

10 Although some uAUGs are recognized and efficiently used as translation start sites, some

11 others are used poorly or not at all, and allow translation of the main ORF. We thus analyzed

Cryptococcus UAUG position and sequence context to see how translation start codons are

specified in these fungi.

We compared the translation efficiency of genes containing only uAUGs close to the TSS to

those with uAUGs far from the TSS. In C. neoformans, 1,627 of the 10,286 uAUGs are

positioned within the first 20 nt of the TL, and 816 uAUG-containing genes have no uAUG

after this position. The presence of one or several uAUGs close to the TSS ( $<20 \mathrm{nt}$ ) has nearly

no effect on translation efficiency, whereas genes containing UAUGs far from the TSS are less

efficiently translated (Figure 2F), and similarly in C. deneoformans (Figure S2.2F).

\section{A Kozak sequence context determines AUG translation initiation.}

To analyze the importance of AUG sequence context for translation initiation in

Cryptococcus we used the $5 \%$ most translated genes (hiTrans $n=330$ ) to construct a 
1 context contains a purine at the -3 position, a hallmark of the Kozak consensus sequence

2 (19). However, there is very little enrichment for the +1 position, in contrast with the

3 mammalian Kozak context in which a $G$ is present in +1 ((A/G)CCAUGG) (19). Because of the

4 limited sequence bias downstream of the AUG, and its confounding with signals of $\mathrm{N}$ -

5 terminal amino acids and codon usage, we do not consider it further. However, we found a

6 slight sequence bias in the positions -10 to -7 that is outside the metazoan Kozak context.

7 We thus calculated "Kozak scores" for all uAUGs against the position weight matrix (pwm) of

8 the Kozak context from -10 from AUG through to AUG (Figure 3A). We compared the Kozak

9 scores of the annotated AUGs (aAUGs) with those of the $5 \%$ most highly translated genes,

10 the first upstream AUG (UAUGs) and the first downstream AUG ( $\left.d_{1} A U G\right)$. Highly translated

aAUGs have a higher score than typical aAUGs, and aAUGs have usually a higher score than

the $u A U G s$ and $d_{1} A U G s$ (Figure $3 B$ ). This suggests that the sequence context of the AUG

codon might be of importance in the selection of the translation start site in Cryptococcus.

We next asked if the sequence context of UAUGs affected their ability to repress translation of the annotated ORF, focusing on transcripts with only a single UAUG. For UAUGs close to the TSS, there is no correlation between UAUG Kozak score and the translation efficiency of the aORF; there is a weak negative correlation if the UAUG is far from the TSS (Figure 3D).

This indicates that the location of an UAUG impacts its activity. The most striking examples of translational repression in Figure 2 tend to have multiple high-score uAUGs (scores

CNAG_06246, u $u_{1} A U G$ 0.93, u $u_{2} A U G$ 0.86; CNAG_03140, u 1 AUG 0.85, u $u_{2} A U G$ 0.76;

CNAG_07813, u 1 AUG 0.79; CNAG_07695, u 1 AUG 0.97, u $\mathrm{u}_{2} A U G$ 0.90).

We also asked if the AUG score affects the AUG usage transcriptome-wide, by comparing the 
1 codon neighbourhood downstream of the $\mathrm{u}_{1} A U G$ and aAUG. We considered the relative

2 occupancy to control for transcript-specific differences in abundance and cap-dependent

3 initiation-complex recruitment. A higher score difference is associated with higher relative

4 ribosome occupancy, while the control comparison with RNA-Seq coverage shows a smaller

5 effect (Figure 3E).

6

$7 \quad$ Nonsense-mediated decay acts on uORF-containing genes.

8 An mRNA molecule translated using an UAUG can be recognized as a premature stop codon

9 bearing molecule and will be as such degraded by the nonsense-mediated mRNA decay

10 (NMD) (41). To test this concept, we first sequenced RNA from $C$. deneoformans strains with

11 the conserved NMD factor Upf1 deleted (33), finding 370 genes with increased mRNA abundance and 270 with decreased (Figure 4A, Table S2; 2-fold difference in levels at $1 \%$ FDR).

We next compared the fold-change in abundance of uORF-containing or uORF-free mRNAs.

Two genes with extreme increases in upf1 $1 \Delta$ are also extremely translationally repressed uORF-containing genes we identified above (Figures 2, S2.1, S2.2): CNF00330 (CNAG_07695 homolog, 11-fold) and CNG04240 (CNAG_03140 homolog, 8-fold). Another extreme is the carbamoyl-phosphate synthase CND01051 (5-fold up in upf1D), a homolog of S. cerevisiae CPA1 and N. crassa arg-2. These orthologs are regulated by a conserved uORF encoding a arginine attenuator peptide that have all been verified to repress reporter gene synthesis in a N. crassa cell-free translation system (42). Consistent with this model, in both $C$. neoformans and $C$. deneoformans the native UORF shows strong ribosome occupancy while the aORF is translationally repressed $(C n T E=0.47, C d T E=0.38$; Figure S4.1). 
1 In general, uORF-containing genes are more likely to be up regulated in the upf1 $\triangle$ mutant

2 than uAUG-free genes (Figure 4B), suggesting that uORFs negatively regulate mRNA

3 abundance in Cryptococcus, in addition to repressing translation of the main ORF. Similarly,

4 uORF-containing genes are enriched for NMD-sensitivity only when the UAUG is more than

$520 \mathrm{nt}$ from the TSS (Figure 4C), suggesting that TSS-proximal uAUGs ( $<20 \mathrm{nt}$ ) are skipped,

6 and generally not used as translation start codons in Cryptococcus.

7 Next, we asked if uAUG Kozak score affects mRNA decay via the NMD pathway. Restricting

8 our analysis to genes with a single UAUG $(n=1,421)$, we binned genes according to their

9 Kozak score. We find that mRNAs that contain uORFs which start with a higher Kozak-score

10 UAUG are more likely to increase in abundance in the upf1 $\triangle$ mutant (Figure 4D). Indeed, the

11 abundance increase is monotonically correlated with the mean of the score bins.

In conclusion, in Cryptococcus, the position and the sequence context of uAUGs determines their usage as translation start codons, and the effect of the uORF on stimulating nonsensemediated decay of the mRNA.

\section{Start codon sequence context and uORF regulation in other fungi}

We then examined sequences associated with translation start codons in other fungi, for which both RNA-Seq and Riboprofiling data were available, and for which the annotation was sufficiently complete (i.e. S. cerevisiae; Neurospora crassa, Candida albicans and Schizosacchomyces pombe). We analyzed the Kozak context associated with aAUG of all annotated coding genes, of the $5 \%$ most translated genes (hiTrans), and for mRNAs encoding cytoplasmic ribosomal proteins (CytoRibo), as a model group of highly expressed and co-regulated genes defined by homology (Table S3). Cytoplasmic ribosomal proteins have informative Kozak contexts, with a strong A-enrichment at the positions -1 to- 3 and 
1 weak sequence enrichment after the AUG in all these species (Figure 5A). The total

2 information content of the Kozak sequence is higher for CytoRibo genes than HiTrans, and

3 higher for HiTrans than all annotated genes, in all these fungi (Figure 5B). Nevertheless,

4 these contexts have also some species specificity: Kozak sequences for HiTrans and CytoRibo

5 are more informative in Cryptococcus and N. crassa than in S. pombe, C. albicans, and S.

6 cerevisiae. In particular, the C-enrichment at positions $-1,-2$ and -5 in Cryptococcus and $N$.

7 crassa is absent in S. cerevisiae, and we observed no sequence enrichment upstream of the -

84 position for S. pombe and very little for S. cerevisiae. In contrast, a $-8 \mathrm{C}$ enrichment, similar

9 to the Cryptococcus pattern, was observed in N. crassa. The $-10-6 \mathrm{~A} / \mathrm{T}$ rich region for $C$.

albicans is likely to reflect an overall A/T-richness of the TLs in C. albicans.

11 The analysis of the TL sequences from these fungi, excluding $C$. albicans for which no TL annotation is available, also shows species specificity. The average TL length in S. cerevisiae (84 nt) is less than half that in Cryptococcus (Figure 5C). In sharp contrast with Cryptococcus, only 985 uAUGs are present in 504 genes, which correspond to $18 \%$ of the genes with an annotated TL in S. cerevisiae. Moreover, the density of the UAUGs is very low and UAUGs have no global effect on TE in this yeast (Figures 5D,E, Fig S5.1).

More broadly, short TLs with very low UAUG density are more the exception than the rule in the fungal kingdom (Figure 5C). Most fungi have large number of UAUGs in their TL and these uAUGs globally down regulate gene expression (Figure S5.1). Our analysis in Cryptococcus, together with the strength and quality of the Kozak contexts associated with the aAUG, suggest that fungi in general are able to discriminate between AUGs with different sequence context, and thus are using AUG sequence context to regulate gene expression at the post transcriptional level. 


\section{Kozak context controls leaky scanning in Cryptococcus}

2 We earlier calculated the Kozak score of the first downstream AUG ( $\left.d_{1} A U G\right)$ within each CDS:

3 these $d_{1} A U G$ scores are mostly lower than the score of the aAUGs (Figure 3B), consistent

4 with most annotations correctly identifying a good-context AUG as the start codon. Yet, we

5 identified number of $d_{1}$ AUGs with a high score $(n=1109$ above 0.826 , the median Kozak score

6 for aAUGs; $n=131$ above 0.926, the median for hiTrans), which could be efficiently used as

7 translation start codon. The scanning model of translation initiation predicts that the $d_{1} A U G$

8 will be used as the start codon only if pre-initiation complexes leak past the aAUG. Thus if

9 the aAUG has a strong sequence context, most ribosomes will start translation there and the

$d_{1}$ AUG will be not used as a translation start codon.

11 To identify potential leaky translation initiation events, we compared the aAUG and $d_{1} A U G$ scores for each of the $50 \%$ most expressed genes (Figure $6 \mathrm{~A}$ ). For above-median aAUG score genes, the score of the $d_{1} A U G s$ can be very high or very low. By contrast, for the genes with a low aAUG score, there is a bias toward higher $d_{1} A U G$ score, suggesting that for these genes the strong $d_{1} A U G$ could be used as alternative translation start site (Figure $6 A$ ).

To test whether AUG score affects translation initiation, we calculated the ratio of ribosome protected fragment density and RNA-Seq density around each aAUG and $d_{1} A U G$, and the difference in score between these two AUGs (Figure 6B). We found a weak positive correlation between the difference in scores of the two AUGs and RNA-Seq density at these specific loci, raising the possibility that transcription start sites sometimes occur downstream of a weak aAUG. By contrast, the relative ribosome density sharply increases at the $d_{1} A U G s$ when the difference in score between the $d_{1} A U G$ and the aAUGs increases. This suggests 
1 that for these genes, both AUGs can be used as translation start codon, as because a subset

2 of scanning ribosomes leak past the aAUG and initiate at the $d_{1} A U G$.

4 Kozak context-controlled scanning specifies alternative N-termini in Cryptococcus and

5 Neurospora

6 We next determined which groups of genes could be affected by potential alternative start

7 codon usage. We focused our analysis on the $50 \%$ most expressed genes for which the

8 difference in score between the aAUG and $d_{1} A U G$ was the highest (difference in wide score

$9 d_{1} A U G-a A U G>0.1, n=167$ for $C$. neoformans) (Table S4). Strikingly, for $66 \%$ of these genes

$10(110 / 167)$ the $d_{1} A U G$ is in frame with the corresponding aAUG, with a median of $69 \mathrm{nt}$ (mean

$1179 \mathrm{nt}$ ) between the two AUGs. Thus, alternative usage of in-frame AUGs would result in

12 proteins with different $\mathrm{N}$-terminal ends. Supporting this hypothesis, $37 \%$ of these proteins

$(41 / 110)$ possess a predicted mitochondrial targeting sequence located between the two

AUGs, far exceeding the $8 \%$ genome-wide (560/6788). This suggests that the usage of the annotated start codon would target the isoform to mitochondria, whereas the usage of the $d_{1} A U G$ would produce a protein specific to the cytoplasm or another organelle. Examples of alternative localization driven by alternative $\mathrm{N}$-termini have been observed across eukaryotes (43).

The pattern of predicted dual-localization, i.e. enrichment of high-score $d_{1} A U G s$ in-frame with predicted mitochondrial localization signal on the longer $\mathrm{N}$-terminal, is conserved in some fungi but not others (Figure 6C). In a null model where coding sequences have random nucleotide content, we would expect roughly $1 / 3$ of $d_{1} A U G$ s to be in frame. In 6 fungal species we examined, for $d_{1} A U G s$ whose score is comparable to or less than the aAUG they 
1 follow, the proportion in frame is close to (Cryptococcus, N. crassa) or less than $1 / 3$. These

2 proportions are similar when we considered reasonably expressed (above-median) or low

3 expressed genes. The pattern differs for proteins with a $d_{1} A U G s$ whose score high relative to

4 the aAUG they follow $\left(\mathrm{d}_{1}\right.$ AUG score $>$ aAUG score +0.1$)$. In Cryptococcus and N. crassa, most

5 reasonably expressed mRNAs are in-frame and over $1 / 3$ of these in-frame high-score $d_{1} A U G s$

6 have predicted mitochondrial localization. In S. cerevisiae and C. albicans, a relative slight

7 enrichment for in-frame $d_{1} A U G s$ and for protein possessing a mitochondrial targeting

8 sequence for high-scoring $d_{1}$ AUGs can be also observed. By contrast, in S. pombe we see

9 depletion in the in-frame/out-of-frame ratio, even in these proteins with high-scoring

$10 d_{1}$ AUGs.

11 These results suggest that the extent to which alternate translation start codons regulate proteome diversity is variable in fungi. Accordingly, we identified a number of Cryptococcus proteins with potential alternative start codons and N-terminal targeting sequences, whose two homologs in S. cerevisiae are known to be necessary in two compartments of the cells. For instance, CnPUS1/CNAG_06353 is an homolog of both the mitochondrial and Cytoplasmic tRNA:pseudouridine synthases encoded by the PUS1 and PUS2 paralogs in S. cerevisiae. In C. neoformans, ribosome occupancy at both the aAUG and $d_{1} A U G$ of CNAG_06353, and the presence of transcription start sites both sides of the aAUG (Figure 6D), argues that both AUGs are used as start codons, and transcription and translation regulation could co-operate to set isoform levels. Similarly, CnGLO1/CNAG_04219 encodes both the cytoplasmic and nuclear isoforms of the glyoxalase I depending of the alternate AUG usage (Figure S6.1B). The next enzyme in this pathway, Glyoxalase II, is likewise encoded by CnGLO2/CNAG_01128, which is a homolog of both cytoplasmic (Glo2) and mitochondrial (Glo4) enzymes in S. cerevisiae. CNAG_01128 has a weak aAUG, strong $d_{1} A U G$, 
1 and $\mathrm{N}$-terminal predicted mitochondrial targeting sequence (Figure $\mathrm{S} 6.1 \mathrm{C}$ ). Finally, we

2 observed that nine members of the amino-acyl tRNA synthetase gene family have predicted

3 alternate localization from alternate AUG start codons.

\section{Amino-acyl tRNA synthetases (aaRSs) are frequently single-copy and dual-localized in}

6 Cryptococcus

7 The tRNA charging activity of aaRSs is essential in both cytosol and mitochondria to support

8 translation in each compartment, and examples of alternative localization of two aaRS

9 isoforms of a single gene have been observed in fungi, plants, and animals (44-46). This

10 implies that a eukaryote with a single genomic homolog of an aaRS is likely to make distinct

11 localized isoforms from that locus. Thus, we examined predicted aaRS localization in fungi.

We assembled gene lists of aaRSs in diverse fungi from homology databases OrthoDB (47) and PANTHERdb (48), adding a mitochondrial SerRS (CNAG_06763/CNB00380) to the list of

Cryptococcus aaRSs analysed by Datt and Sharma (49).

In $C$. neoformans and $C$. deneoformans, eleven aaRSs are each expressed from a single

genomic locus, including the homologs of all five S. cerevisiae aaRSs whose dual-localization has been verified (Table S5). Nine of these Cryptococcus aaRSs have the same structure of a poor-context annotated AUG followed by a predicted mitochondrial targeting sequence and a strong-context $d_{1} A U G$ (Figure 7A/B; AlaRS, CysRS, GlyRS, HisRS, ValRS, LysRS, ProRS, ThrRS, TrpRS). The similar annotated AUG contexts, sharing an unfavourable $-3 U$, suggests that the same mechanism could lead to leaky translation initiation at most of these (Table S6). At the downstream AUGs, the strong Kozak context is consistent with efficient translation initiation of the cytoplasmic isoform from this start codon (Table S6). 
1 The two remaining single-copy aaRSs have near-AUG translation initiation sites upstream of

2 predicted mitochondrial targeting sequences. Translation of ArgRS starts at an AUU codon

3 with otherwise strong context (cccaccAUU) conserved in both Cryptococcus species. This N-

4 terminal extension includes a predicted mitochondrial targeting sequence (mitofates $p>$

$5 \quad 0.95$ for both species). Translation of LeuRS starts at adjacent ACG and AUU codons which

6 collectively provide strong initiation context (gccaccACGAUU in C. neoformans, gccACGAUU

7 in $\mathrm{C}$. deneoformans). This $\mathrm{N}$-terminal extension also includes a predicted mitochondrial

8 targeting sequence (mitofates $p \approx 0.7$ for both species).

9 In Cryptococcus, alternative aaRS isoforms appear to be mostly generated by alternative

10 translation from a single transcript, and sometimes by alternative transcription start sites.

11 On all the predicted dual-localized aaRSs, we observe ribosomal occupancy starting at the earliest start codon (Figure 7C/D, Fig S7.1). LysRS/CNAG_04179 contains only a single cluster of transcription start sites, upstream of the aAUG (Figure 7C). ProRS/CNAG_04082 contains a wider bimodal cluster of TSSs, both upstream of the aAUG. Similarly, most transcription initiation is well upstream of the aAUG in CysRS/CNAG_06713, LeuRS/CNAG_06123, ThrRS/CNAG_06755, and ValRS/CNAG_07473. However, for GlyRS/CNAG_05900, and HisRS/CNAG_01544, we observe alternative transcription start sites closely upstream of the annotated start codon, that are likely to affect the efficiency of start codon usage. In ArgRS/CNAG_03457 there is also an alternative transcription start site, close to the nearAUG start codon for the mitochondrial form. In AlaRS/CNAG_05722 and TrpRS/CNAG_04604 we detect some transcription start sites between the alternative start codons, and TrpRS also has an uORF in the transcript leader that is likely to affect translation. These 
1 regulated largely by start codon choice. For some genes this regulation is backed up by

2 alternative TSS usage.

3 Some dual-localized genes use an upstream near cognate codon (DualNCC) in all these fungi,

4 but the NCC-initiated aaRS are not the same from one fungus to the other. For instance,

5 both Cryptococcus and N. crassa AlaRS use DualAUG whereas in S. pombe, S. cerevisiae and

6 C. albicans a DualNCC is used. On the other hand, S. pombe GlyRS is regulated by DualNCC

7 whereas the other ones use a DualAUG regulation. Substitution between weak AUG codons

8 and near-cognate codons seems thus to have taken place multiple times in the fungal

9 kingdom.

\section{Amino-acyl tRNA synthetases as an evolutionary case study}

To understand patterns of dual-localization, we next examined the evolution of aaRSs. The ancestral eukaryote is thought to have had two complete sets of aaRS, one mitochondrial and one cytoplasmic, but all mitochondrial aaRSs have been captured by the nuclear genome and many have been lost (50). Thus we examined aaRS phylogenetic trees in more detail. For some amino acids (Asn, Asp, Glu, Iso, Met, Phe, Ser, Tyr), reference fungi have distinct cytoplasmic and mitochondrial aaRSs that cluster in separate trees (51). We also do not consider Gln, because organellar Gln-tRNA charging in some species is achieved by an indirect pathway (52).

Dual-localized AlaRS, CysRS, and HisRS in the 6 fungi we focus on are each monophyletic

(51). Even these aaRS can be encoded by two genes in some other fungi: AlaRS is duplicated to one exclusively mitochondrial and another exclusively cytoplasmic gene in the Saccharomycete yeast Vanderwaltozyma polyspora (53). For CysRS, Aspergillus versicolor (ASPVEDRAFT_141527 and ASPVEDRAFT_46520) and Coprinus cinerea (CC1G_03242 and 
1 CC1G_14214) have two copies, one of which has a predicted mitochondrial targeting

2 sequence. For HisRS, Rhizopus delemar (RO3G_01784 and RO3G_16958) and Phycomyces

3 blakesleeanus (PHYBL_135135 and PHYBL_138952) likewise contain gene duplications.

4 Similarly, S. cerevisiae has two ArgRS genes that arose from the whole-genome duplication:

$5 \quad$ RRS1/YDR341C is essential, abundant, and inferred to be cytoplasmic (54) while

6 MSR1/YHR091C has a mitochondrial localization sequence and MSR1 deletions have a petite

7 phenotype (55), although both have been detected in mitochondria suggesting some

8 residual dual-localization of the cytoplasmic enzyme (56). The second S. cerevisiae stress-

9 responsive cytoplasmic copy of GlyRS also arose from the whole-genome duplication (57). S.

10 pombe cytoplasmic VaIRS is monophyletic with dual-localized ValRS in other fungi, and

11 Schizosaccharomyces also has a paralogous but diverged mitochondrial ValRS that appears

12 to be descended from an early eukaryotic ValRS of mitochondrial origin (58).

LysRS appears to have been duplicated in an ancestor of ascomycetes: ascomycete

mitochondrial homologs cluster together, and ascomycete cytoplasmic homologs cluster together, while the single basidiomycete homolog clusters close to the base of this split from other opisthokonts (51). By contrast, LeuRS, ProRS, and TrpRS are each represented by two distinct proteins in ascomycetes, one cytoplasmic and one mitochondrial and of independent descent, but the mitochondrial homolog has been lost in Cryptococcus species. In basidiomycetes Ustilago and Puccinia, homologs of mitochondrial LeuRS and ProRS are not present, but there is a homolog of mitochondrial TrpRS; all these have a single homolog of the cytoplasmic TrpRS (51). Our independent phylogenetic analysis of LysRS and ProRS agrees with the conclusions from PANTHERdb (Figures 7E/F). These analyses show that aaRSs have undergone multiple incidences of at least two processes during fungal evolution: 
1 losses associated with the dual-localization of the remaining gene, and duplications followed

2 by specialization.

3

4 Evolutionary conservation of gene-specific feedback regulation by alternate AUG usage

5 We also observed striking examples of gene-specific regulation by start codon context in

6 Cryptococcus, in translation factors affecting start codon selection, supporting previously

7 proposed models of feedback regulation $(59,60)$.

8 Translation initiation factor elF1, which enforces selection of strong context start codons, is

9 encoded by an mRNA with poor start codon context in diverse eukaryotes, driving an

autoregulatory program $(59,61)$. In C. neoformans, elF1 also initiates from a poor-context

cuuaguugaAUG start (score 0.75), and ribosome profiling reads are spread across the

annotated ORF (Figure 8A). Intriguingly, the next AUG is out-of frame and has strong context

cuccaaaaAUG (score 0.98), with a same-frame stop codon 35 codons later, suggesting that

this could represent a downstream short ORF that captures ribosomes that have leaked past

the poor-context start. To test this hypothesis, we examined the $5^{\prime}$ ends of riboprofiling

reads, which report on the translation frame of the ribosomes (36). Riboprofiling reads from

the $5^{\prime}$ and $3^{\prime}$ of the elF1 annotated ORF are roughly $77 \%$ in frame $0,10 \%$ in +1 , and $13 \%$ in

+2 , as are reads on two other highly expressed genes, eEF1 $\alpha$ and HSP9O. By contrast, in the

hypothesized downstream ORF, reads are only $57 \%$ in frame $0,32 \%$ in frame +1 , and $11 \%$ in

frame +2 , consistent with translation occurring in both frame 0 and +1 . The gene structure is

conserved in $C$. deneoformans, with a weak aAUG (score 0.76), a strong $d_{1} A U G$ (score 0.98)

in the +1 frame, followed by an enrichment in +1 -frame riboprofiling reads (Figure S8.1A,B).

We observe small increases in elF1 mRNA levels in the upf1 $\Delta$ strain of $C$. deneoformans at

both $30^{\circ} \mathrm{C}(1.16 \mathrm{x}, \mathrm{p}=0.04)$ and $37^{\circ} \mathrm{C}(1.09 \mathrm{x})$, so NMD could regulate this transcript. Overall, 
1 our data support the hypothesis that the downstream ORF of elF1 is translated after read-

2 through of the annotated AUG, and that the downstream ORF contributes to translation

3 regulation of the annotated ORF.

4 Translation initiation factor elF5 reduces the stringency of strong-context start-codon

5 selection, and is encoded by an mRNA with a repressive UORF initiated from a poor-context

6 uAUG in diverse eukaryotes (60). In Cryptococcus, elF5 (TIF5/CNAG_01709) also contains a

7 UAUG with the poor sequence context aaagaguucAUG (score 0.72), while the main ORF of

8 elF5 is initiated by a strong context cccgcaaaAUG (score 0.94). We detect ribosomal density

9 on the UORF of TIF5 comparable to that on the main ORF (Figure $8 C$ ), suggesting there is

10 substantial translation initiation at the UAUG. There is also clear translation initiation at a

11 further upstream near-cognate CUG codon. The gene structure is conserved in $C$.

deneoformans TIF5, with the same pattern of riboprofiles at upstream poor-context AUG

and near-cognate codons (Figure S8). Further, the $C$. deneoformans homolog transcript

abundance increases substantially in the upf1 1 strain $\left(2.6 \mathrm{x}, \mathrm{p}<10^{-50}\right)$. This supports the

model that elF5 translation is repressed by upstream reading frames initiated from poor

start codons, leading to nonsense-mediated decay of the transcript.

These examples further illustrate that the first start codon is not always used, but rather

start codon usage is driven by the sequence context, and that variability in start codon

context including the canonical AUG sequence is used for translational regulation.

\section{Variable inserts in eTIFs correlate with variation in translation initiation determinants}

The conserved proteins elF1, elF5, and elF1A play pivotal roles in start codon selection in S. 
1 variability in start codon preference is linked to these initiation factors, we generated

2 multiple sequence alignments of their homologs in fungi.

3 Translation initiation factor elF1 shows striking sequence variation across fungi, notably at

4 multiple Cryptococcus-specific sequence insertions that result in a 159-aa protein

5 substantially larger than the 108-aa S. cerevisiae homolog (Figure 9A). Variation in elF1

6 occurs at and around positions known to modulate start codon selection in S. cerevisiae (61).

7 For instance, a T15A substitution increases fidelity in ScelF1 (61), and an analogous T15A

8 substitution is present in elF1s from Neurospora and other filamentous fungi, while both

9 Cryptococcus homologs have the T15V substitution. The three fungi that tend not to use

10 alternative AUG start codons in the regulation of proteome diversity, S. cerevisiae, $C$.

11 albicans, and S. pombe, all have a threonine residue at position 15. Variation in fungal elF1

extends far beyond this $\mathrm{N}$-terminal region: similar patterns of sequence diversity occur at

the positions E48, L51, D61 that have been shown to increase fidelity in ScelF1 (61). By

contrast, positions $\mathrm{K} 56, \mathrm{~K} 59, \mathrm{D} 83, \mathrm{Q} 84$, at which mutations have been shown to reduce

fidelity in ScelF1 (61), are highly conserved in fungi.

We next tested how the translation pre-initiation complex could be affected by the

The $\mathrm{N}$-terminal insertion is not visible in the structure, but could be close to the acceptor

arm of tRNAi. The N-proximal loop insertion of CnelF1 extends from the ScelF1 sequence 
1 codons and weak AUG codons (63). CnelF1 loop 2 has substitutions at both these

2 functionally important sites, and is extended by a further 14 hydrophobic and negative

3 residues. The last insertion in CnelF1 extends a loop facing the solvent-exposed surface of

4 ScelF1. Collectively, this shows that there are likely major differences in the elF1-tRNAi

5 interaction surface in Cryptococcus relative to other fungi, an interaction critical for start

6 codon selection (63).

7 The N-terminal domain of elF5 (elF5-NTD) replaces elF1 upon start codon recognition, and

8 we found between-species variation in CnelF5 at tRNAi interaction surfaces corresponding to

9 variability in CnelF1 (Figure 9C, S9.1A). ScelF5 Lys71 and Arg73 in loop 2 make more

10 favourable contacts with the tRNAi than the corresponding residues of ScelF1, so that the

11 shorter loop 2 of ScelF5 may allow the tRNAi to tilt more towards the 40 S subunit (27).

Although Arg73 is conserved across fungi, Lys71 is absent in CnelF5 loop 2 (67-SMAN-70), which is two amino acids shorter than ScelF5 loop 2 (66-SISVDK-71). Collectively, the longer loop 2 of CnelF1 and the shorter loop 2 of CnelF5 suggest that the conformational changes accompanying start codon recognition may be more exaggerated in Cryptococcus, providing a mechanistic hypothesis for stronger genomic patterns of start codon recognition.

Fungal elF1A homologs also diverge from ScelF1A at regions that modulate translation initiation fidelity (Figure S9.1B), for example the N-terminal element DSDGP (61). The and along with other basidiomycetes lacks a loop at ScelF1A positions $135-149$. This Cterminal region of ScelF1A contributes to pre-initiation complex assembly and binds elF5B (64) and elF5 (65), and domain deletions or local alanine substitutions reduce fidelity of translation start site selection $(61,64,66)$. 
1 Thus, although structural analysis of the Cryptococcal initiation complex will be required for

2 a detailed mechanistic understanding, our initial analysis suggests that sequence variability

3 in fungal elFs could plausibly account for differences in start codon selection between

4 different species.

5 


\section{DISCUSSION}

2

3 Our annotation of transcript structure and translation in two pathogenic Cryptococcus

4 species and our analysis of published data from other species show that start codon context

5 has a major effect on protein production, regulation, diversity, and localization in the fungal

6 kingdom. As such this work represents a useful resource for the field. While the genome-

7 wide effect of start codon context is weak in S. cerevisiae (20), we find that other fungi, from

8 Neurospora to Cryptococcus, use start codon context to regulate translation initiation to a

9 far greater extent. These fungi have long and AUG-rich TLs, and more information-rich and

10 functionally important Kozak sequences. Further, Cryptococcus and Neurospora display extensive evidence of leaky scanning of weak AUG codons that is used for regulation by upstream ORFs and to generate alternate N-terminal isoforms with different subcellular localization.

\section{Widespread leaky scanning controlled by start codon context in $C$. neoformans}

Translation initiation regulation can be enabled by start codons that are imperfectly used, so that scanning pre-initiation complexes can leak past them. According to the scanning model of translation initiation, a "perfect" strong start codon would prevent this by capturing all the scanning PICs, and leave none for regulatable downstream initiation. For example, the downstream out-of-frame ORF of Cryptococcus elF1 is likely to be translated only by PICS that leak past the annotated AUG. The alternative second in-frame AUG of dual-localized proteins is also initiated only by PICS that have leaked past the initial AUG. Our data show this leakiness-driven dual-localization is common in Cryptococcus, in addition to being conserved across eukaryotes in gene classes such as tRNA synthetases. Our data also argue 
1 that AUGs that are proximal to the 5' cap, or that have poor sequence context, are

2 commonly leaked past in Cryptococcus, as shown previously in studies of yeast (67) and

3 mammals $(17,68)$. We note that leakiness-driven translation regulation is not the only

4 mechanism regulating alternative translation from a single mRNA and is distinct from those

5 that depend on either blocking scanning, or on recycling of post-termination ribosomes such

6 as in the case of S. cerevisiae GCN4 (37).

7

\section{Functional role of start codon context varies across the fungal kingdom}

9 Cryptococcus and Neurospora have long TLs that are AUG-rich, and extended start codon

context sequences that suggest a higher ability to discriminate against poor-context AUGs.

Several lines of evidence argue that efficiency with which upstream AUGs capture initiation

complexes is determined by the AUG sequence context. The most spectacular examples of

uORF-associated translation repression in Cryptococcus are associated with good-context

uAUGs with high ribosome occupancy. However, such strong-context high-occupancy uAUGs

are rare. In Cryptococcus and Neurospora, the leakiness of potential AUG translation start

sites is also extensively used to diversity the proteome by alternative $\mathrm{N}$-terminal formation.

In comparison, S. cerevisiae, S. pombe and C. albicans appear to be less efficient in

discriminating AUGs based on their sequence context. S. cerevisiae has minimized the

possibility of regulation of gene expression by uORFs: it has unusually short TLs, these TLS

are unusually AUG-poor, uAUGs tend to have poor context, and there is no statistical studies $(21,22)$ and classic examples such as GCN4 show that uAUGs can repress translation 
1 transcript leaders that contain repressive uAUGs (“long undecoded transcript isoforms") are

2 more common during alternative growth conditions for this yeast. Moreover, in S. cerevisiae,

3 near-cognate codons appear to be more common starts for alternative $\mathrm{N}$-terminal formation

4 (71). This suggests that leaky scanning from near-cognate codons, more than from AUGs,

5 might be an important mode of regulation in S. cerevisiae. The situation is different in S.

6 pombe, which has long AUG-rich TLs but is depleted for downstream in-frame AUGs.

7 Consequently, uAUGs globally repress gene expression, but do not appear to regulate

8 alternative protein production through alternative AUG start codons. We speculate that the

9 comparatively uninformative Kozak context in S. pombe might be variable enough to

10 regulate translation initiation rate but not proteome diversity.

11 We found that multiple near-cognate start codons are used for leaky initiation in

Cryptococcus: ACG for the mitochondrial isoform of LeuRS, AUU for the mitochondrial

isoform of ArgRS, and the upstream CUG in elF5. Further work will be needed to quantify the

extent of near-cognate start codon usage in Cryptococcus in different growth conditions and compare it to other organisms $(14,72)$.

\section{Leaky scanning through weak AUGs could regulate the mitochondrial proteome}

We computationally predicted dozens of dual-localized proteins with alternative start

codons that confer an $\mathrm{N}$-terminal mitochondrial targeting sequence in their longest isoform.

We did not identify enrichment of proteins with predicted dual-localization in the cytoplasm

and in the nucleus, or with a signal peptide followed by an alternative start codon (data not isoforms of dozens of proteins. 
1 Mechanisms to control initiation efficiency of a mitochondrial-localized regulon could

2 include intracellular magnesium concentration (73), variations in availability or modification

3 status of shared initiation factors, variations of the ratio of mitochondrial volume to

4 intracellular volume (74), or specialized factors to promote initiation specifically of

5 mitochondrial isoforms with their specialized start codon context. Nakagawa et al (75)

6 previously suggested that distinct Kozak contexts might be recognized by different molecular

7 mechanisms.

8 One candidate mechanism involves the translation initiation factor 3 complex, which has a

9 role in regulating the translation initiation of mitochondrial-localized proteins across

eukaryotes. In S. pombe, subunits elF3d/e promote the synthesis of mitochondrial electron

transfer chain proteins through a TL-mediated mechanism (76). In S. cerevisiae and

Dictyostelium discoideum the conserved elF3-associated Clu1/CluA protein affects mitochondrial morphology (77), and the mammalian homolog CLUH binds and regulates mRNAs of nuclear-encoded mitochondrial proteins $(78,79)$. Metazoans have 12 stablyassociated subunits of elF3, which are conserved in most fungi including N. crassa (80), Cryptococcus, and the Saccharomycetale yeast Yarrowia lipolytica (Table S7). Interestingly, species that tend not to use alternate AUG codons for dual-localization have lost elF3 subunits: elF3d/e/k/l/m are lost in C. albicans, and additionally elF3f/h in the related $S$. cerevisiae; S. pombe has independently lost elF3k/I (Table S7; (51)). Further work will be needed to investigate the role of elF3 in regulating mitochondrial- and dual-localized proteins in the fungal kingdom. 
1 Selection on genome compaction in unicellular yeasts, which has independently led to gene

2 loss and high gene density in multiple lineages of yeast, could lead to shorter TLs. However,

3 Saccharomyces, Schizosaccharomyces, and Cryptococcus have all independently evolved

4 yeast lifestyles with compact genomes, yet their average TL lengths differ three-fold.

5 Mutations in gene expression machinery, such as the variation in elF1 noted above, would

6 alter selective pressure on start codon context, and thus UAUG density. Cells have multiple

7 redundant quality control mechanisms, and flexible protein production through leaky

8 scanning could be buffered by such mechanisms enabling their evolution. Key control

9 mechanisms acting on mRNA, such as RNAi and polyuridylation, have been lost in fungal

10 lineages such as Saccharomyces, which might explain their more 'hard-wired' mechanism of

11 translation initiation.

Unexpectedly, highly conserved core translation initiation factors, such as elF1, have

distinctive sequence inserts in Cryptococcus that are not shared even by basidiomycetes

such as Puccinia and Ustilago. One possibility is genetic conflict, as genetic parasites hijack

the gene expression machinery (81). Thus, the unique aspects of the Cryptococcus

translation initiation machinery could have arisen from a past genetic conflict in which rapid evolution of initiation factors in an ancestor enabled evasion of a genomic parasite (e.g. a mycovirus) that would otherwise hijack initiation.

\section{Data Availability}

Raw and summarized sequencing data are available on GEO under accession numbers GSE133695 (RNA-seq, TSS-seq, PAS-seq) and GSE133125 (ribosome profiling and matched RNA-seq).

\section{Acknowledgments}


We thank members of the Wallace, Janbon, and Madhani labs for helpful discussions and comments on the manuscript. We thank Juan Mata for sharing intermediate data related to (Duncan and Mata 2017). We are grateful to J. Weissman (UCSF) for advice on ribosome profiling. Work in the Madhani lab is supported by grants from the US National Institutes of Health. H.D.M. is an Investigator of the Chan-Zuckerberg Biohub. E.W.J.W. is a Sir Henry Dale Fellow, supported by a Sir Henry Dale Fellowship jointly funded by the Wellcome Trust and the Royal Society (Grant Number 208779/Z/17/Z). L.T. is supported by a Wellcome-University of Edinburgh ISSF3 award.

\section{Material and Methods}

\section{DNA and RNA purification, sequencing library preparation}

C. neoformans strain $\mathrm{H} 99$ and C. deneoformans strain JEC21 and were grown in YPD at $30^{\circ} \mathrm{C}$ or $37^{\circ} \mathrm{C}$ under agitation up to exponential or early stationary phase as previously described (32). Briefly, early stationary phase was obtained after $18 \mathrm{~h}$ of growth (final $\mathrm{OD}_{600}=15$ ) starting from at $\mathrm{OD}_{600}=0.5$. C. deneoformans strain NE579 (upf1 $\triangle$ ) (33) was grown in YPD at $30^{\circ} \mathrm{C}$ under agitation in exponential phase. Each Cryptococcus cell preparation was spiked in with one tenth (OD/OD) of S. cerevisiae strain FY834 (82) cells grown in YPD at $30^{\circ} \mathrm{C}$ in stationary phase. Cells were washed, snap frozen and used to prepare RNA and total DNA samples as previously described (32). Each condition was used to prepare biological triplicate samples.

For RNA-Seq, strand-specific, paired-end cDNA libraries were prepared from $10 \mu \mathrm{g}$ of total RNA by polyA selection using the TruSeq Stranded mRNA kit (Illumina) according to manufacturer's instructions. cDNA fragments of $\sim 400$ bp were purified from each library and confirmed for quality by Bioanalyzer (Agilent). DNA-Seq libraries were prepared using the kit TruSeq DNA PCR-Free (Illumina). Then, 100 bases were sequenced from both ends using an Illumina HiSeq2500 instrument according to the manufacturer's instructions (Illumina). 
1 TSS-Seq libraries preparations were performed starting with $75 \mu \mathrm{g}$ of total RNA as previously

2 described (34) replacing the TAP enzyme by the Cap-clip Pyrophosphatase Acid (TebuBio).

3 For each Cryptococcus species we also constructed a control “no decap" library.

4 Briefly, for these control libraries, poly A RNAs were purified from $75 \mu \mathrm{g}$ of RNA from

5 Cryptococcus and $75 \mu \mathrm{g}$ of RNA S. cerevisiae before being dephosphorylated using Antarctic

6 phosphatase. Then, S. cerevisiae RNAs and one half of the RNAs extracted from Cryptococcus

7 were treated with Cap-clip Pyrophosphatase Acid enzyme. The second half of Cryptococcus

8 RNAs was mock treated. Each half of Cap-clip Pyrophosphatase Acid Cryptococcus RNA

9 samples was mixed with the same quantity of S. cerevisiae Cap-clip Pyrophosphatase Acid

10 treated RNAs. The subsequent steps of the library preparation were identical to the

11 published protocol (34). Single 50 bases single end reads were obtained using an an Illumina

HiSeq2500 instrument according to the manufacturer's instructions (Illumina).

For QuantSeq 3'mRNA-Seq preparation we followed the manufacturer instructions (Lexogen

$\mathrm{GmbH}$, Austria). 100 base single end reads were obtained using an Illumina HiSeq2000 instrument according to the manufacturer's instructions (Illumina).

\section{Sequencing data analyses}

For TSS analysis we kept only the reads containing both the oligo 3665

(AGATCGGAAGAGCACACGTCTGAAC) and the 11NCGCCGCGNNN tag (34). These sequences

were removed and the trimmed reads were mapped to the Cryptococcus genome and $\mathrm{S}$. replicates. Their coverage was normalized using the normalization factor used for spiked in 
1 RNA-Seq. TSS positions were then clustered per condition. As most of the observed TSS sites

2 appeared as clusters, we grouped them into clusters by allowing an optimal maximum intra-

3 cluster distance (at $50 \mathrm{nt}$ ) between sites as previously used (34). We then removed the false

4 TSS clusters using the "no-cap" data keeping the clusters i for which

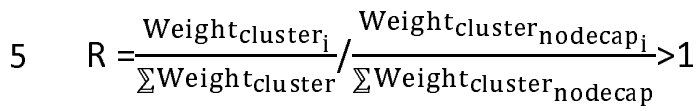

6

7 Similarly, QuantSeq 3'mRNA-Seq reads containing both the Sequencing and indexing primers

8 (Lexogen) were sorted. The reads were then cleaned using cutadapt/1.18 (84) and trimmed

9 for polyA sequence in their 3'end. PolyA untrimmed and trimmed reads were mapped to the

10 adapted Cryptococcus and to the S. cerevisiae genomes with Tophat2 (83) with the same

11 setting as for RNA-Seq. To eliminate the polyadenylated reads corresponding to genomic

polyA stretches, we considered only the reads that aligned to the genomes after polyA

trimming but not before the trimming. The 3 'end position of these reads were considered as

potential PAS. As for the TSS, for each condition we kept only the positions that were

present in all three replicates. Similarly, the PAS dataset was normalized using the spike in

normalization factor and the PAS positions were clustered using the same strategies.

\section{Ribosome profiling and matched mRNA-seq}

Ribosome profiling was performed on both $C$. neoformans $\mathrm{H} 99$ and $C$. deneoformans JEC21,

two biological replicates of WT-H99 and one replicate each of H99 ago1 4 and H99 gwo1A

strains from (30), and one replicate each of WT-JEC21 and JEC21 ago14. There was 
1 Cells were grown to exponential phase in $750 \mathrm{~mL}$ of YPAD with shaking at $30^{\circ} \mathrm{C} .100 \mathrm{ug} / \mathrm{ml}$

2 cycloheximide (Sigma) (dissolved in 100\% ethanol) was added to the culture and incubated

3 for 2 minutes. $50 \mathrm{~mL}$ of the culture was withdrawn for performing RNA-Seq in parallel. Cells

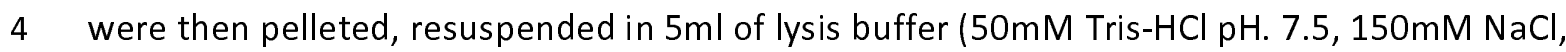

$510 \mathrm{mM} \mathrm{MgCl} 2,5 \mathrm{mM} \mathrm{DTT}, 0.5 \%$ Triton and $100 \mathrm{ug} / \mathrm{mL}$ cyclohexamide) and snap frozen. Lysis,

6 clarification, RNasel digestion, sucrose gradient separation and monosome isolation was

7 performed as previously described (36).

8 Ribosome protected fragments were isolated from the monosome fraction using hot phenol.

9 150ug of the total RNA extracted from the $50 \mathrm{ml}$ of culture in parallel was polyA selected using the Dynabeads mRNA purification kit (Thermo Fisher Scientific) and digested using freshly made fragmentation buffer (100mM NaCO3 pH. 9.2 and 2mM EDTA) for exactly 20 mins.

RNA was resolved on a 15\% TBU gel. A gel slab corresponding to $28-34$ nt was excised for footprint samples and around $50 \mathrm{nt}$ for mRNA samples, then eluted and precipitated. Sequencing libraries were generated from the RNA fragments as described in Dunn et al. with the following modifications (85). cDNA was synthesized using primer oCJ11 (Table S8). Two rounds of subtractive hybridization for rRNA removal was done using oligos ras1-8 listed in Table S8. After circularization Illumina adaptors were added through 9 cycles of PCR. Libraries were sequenced on a HiSeq 2500 (Illumina).

\section{Ribosome profiling data analysis} and then analyzed essentially with the RiboViz pipeline v.1.1.0 (86). In brief, sequencing adapters were removed with cutadapt (84), and then reads aligned to rRNA were removed 
1 by alignment with hisat2 (87). Cleaned non-rRNA reads were aligned to (spliced) transcripts

2 with hisat2 (87), sorted and indexed with samtools (88), and then quantified on annotated

3 ORFs with bedtools (89), followed by calculation of transcripts per million (TPM) and quality

4 control with R (90) scripts included in RiboViz. The cleaned non-rRNA reads were also aligned

5 to the genome with hisat2, and processed analogously, then used to generate figures of

6 genome alignments using ggplot2 (91) in R (90).

\section{Data analysis and visualization}

8 Data analysis and visualization were scripted in R (90), making extensive use of dplyr (92),

9 ggplot2 (91), and cowplot (93). Sequence logos were prepared in ggseqlogo (94). Analysis of

10 differential expression for upf1 $\Delta$ data was performed in DeSeq2 (95). Figures were

11 assembled and annotated in Inkscape v0.92 (https://inkscape.org).

12 Protein sequences were aligned using muscle (96), with default parameters for protein sequences and 100 iterations. Phylogenetic trees were constructed using ClustalW2 tool v2.1 (97) by using the neighbor-joining method with 1000 bootstrap trial replications.

Structural figures were prepared in PyMOL (Schrodinger).

\section{External datasets}

N. crassa (strain OR74A) ribosome profiling data from ((14), GEO:GSE97717) was used to generate highly-translated genes, and ribosome profiling and RNA-seq data from ((98), GEO: GSE71032) used to estimated TE. In both cases, we estimated TPMs using the RiboViz

21 pipeline as above, using the NC12 genome annotation downloaded from EnsemblGenomes (99). TL sequences were also obtained from NC12. 
1 S. pombe (strain 972h) ribosome profiling and RNA-seq data are from (100), and the authors

2 provided us with a table of RPKMs for all replicates as described. Genome sequence and

3 annotation ASM294v2, including TL annotation, were downloaded from EnsemblGenomes

4 (99).

5 C. albicans (strain SC5314) ribosome profiling and RNA-seq data are from (101),

6 GEO:GSE52236), processed with the RiboViz pipeline as above using the assembly 22 of the

7 strain SC5414 genome annotation from CGD (102).

8 S. cerevisiae (strain S288C/BY4741) highly-translated genes use the RPKM table from ((103),

9 GEO:GSE59573), and highly-expressed genes use (104). For TE estimates, we used matched

10 ribosome profiling and RNA-seq estimates from (105), although we did not use this for the

11 list of highly translated genes because near-duplicate paralogous ribosomal protein genes

12 were not present in the dataset, which thus omits a substantial fraction of highly-translated

13 genes. TL sequences were downloaded from SGD (106)

14 Protein homolog lists were assembled with OrthoDB (47) and PANTHERdb (48), with

15 reference to FungiDB (107). The list of cytoplasmic ribosomal proteins was assembled in S.

16 cerevisiae based on (108) with help from SGD (106), extended to other fungi with

17 PANTHERdb (47), and manually curated. 


\section{References}

1. Grigoriev IV, Nikitin R, Haridas S, Kuo A, Ohm R, Otillar R, et al. MycoCosm portal: gearing up for 1000 fungal genomes. Nucleic Acids Res. 2014;42 (Database issue):D699-D704.

2. Fan G, Sun Q, Li W, Shi W, Li X, Wu L, et al. The global catalogue of microorganisms 10K type strain sequencing project: closing the genomic gaps for the validly published prokaryotic and fungi species. GigaScience. 2018;7(5).

3. Shen X-X, Opulente DA, Kominek J, Zhou X, Steenwyk JL, Buh KV, et al. Tempo and Mode of Genome Evolution in the Budding Yeast Subphylum. Cell. 2018;175(6):1533-45.e20.

4. Butler G, Rasmussen MD, Lin MF, Santos MAS, Sakthikumar S, Munro CA, et al. Evolution of pathogenicity and sexual reproduction in eight Candida genomes. Nature. 2009;459:657.

5. Dujon B, Sherman D, Fisher G, Durrens P, Casaregola S, Lafontaine I, et al. Genome evolution in yeasts. Nature. 2004;430:35-44.

6. Stajich JE. Fungal Genomes and Insights into the Evolution of the Kingdom. Microbiology spectrum. 2017;5(4):10.1128/microbiolspec.FUNK-0055-2016.

7. Stajich JE, Dietrich FS, Roy SW. Comparative genomic analysis of fungal genomes reveals intron-rich ancestors. Genome Biol. 2007;8:R223.

8. Coletta A, Pinney JW, Solís DYW, Marsh J, Pettifer SR, Attwood TK. Low-complexity regions within protein sequences have position-dependent roles. BMC systems biology. 2010;4:43-.

9. Goffeau A, Barrell BG, Bussey H, Davis RW, Dujon B, Feldmann H, et al. Life with 6000 Genes. Science. 1996;274(5287):546.

10. Haas BJ, Zeng Q, Pearson MD, Cuomo CA, Wortman JR. Approaches to Fungal Genome Annotation. Mycology. 2011;2(3):118-41.

11. Fervers $\mathrm{P}$, Fervers $\mathrm{F}$, Makałowski W, Jąkalski M. Life cycle adapted upstream open reading frames (uORFs) in Trypanosoma congolense: A post-transcriptional approach to accurate gene regulation. PLOS ONE. 2018;13(8):e0201461.

12. Duncan CDS, Rodríguez-López M, Ruis P, Bähler J, Mata J. General amino acid control in fission yeast is regulated by a nonconserved transcription factor, with functions analogous to Gcn4/Atf4. Proceedings of the National Academy of Sciences of the United States of America. 2018;115(8):E1829-E38.

13. Sundaram A, Grant CM. A single inhibitory upstream open reading frame (uORF) is sufficient to regulate Candida albicans GCN4 translation in response to amino acid starvation conditions. RNA (New York, NY). 2014;20(4):559-67.

14. Ivanov IP, Wei J, Caster SZ, Smith KM, Michel AM, Zhang Y, et al. Translation Initiation from Conserved Non-AUG Codons Provides Additional Layers of Regulation and Coding Capacity. mBio. 2017;8(3):e00844-17.

15. von Arnim AG, Jia $Q$, Vaughn JN. Regulation of plant translation by upstream open reading frames. Plant Science. 2014;214:1-12.

16. Barbosa C, Peixeiro I, Romão L. Gene expression regulation by upstream open reading frames and human disease. PLoS Genet. 2013;9(8):e1003529-e.

17. Kozak M. Point mutations define a sequence flanking the AUG initiator codon that modulates translation by eukaryotic ribosomes. Cell. 1986;44(2):283-92.

18. Kozak M. An analysis of 5'-noncoding sequences from 699 vertebrate messenger RNAs.

Nucleic acids research. 1987;15(20):8125-48.

19. Hinnebusch AG, Ivanov IP, Sonenberg N. Translational control by 5? eukaryotic mRNAs. Science. 2016;352(6292):1413-6.

20. Dever TE, Kinzy TG, Pavitt GD. Mechanism and Regulation of Protein Synthesis in Saccharomyces cerevisiae. Genetics. 2016;203(1):65-107.

21. Dvir S, Velten L, Sharon E, Zeevi D, Carey LB, Weinberger A, et al. Deciphering the rules by which 5'-UTR sequences affect protein expression in yeast. Proceedings of the National Academy of Sciences of the United States of America. 2013;110(30):E2792-E801. 
22. Cuperus JT, Groves B, Kuchina A, Rosenberg AB, Jojic N, Fields S, et al. Deep learning of the regulatory grammar of yeast 5 ' untranslated regions from 500,000 random sequences. Genome Res. 2017;27(12):2015-24.

23. Chen S-J, Lin G, Chang K-J, Yeh L-S, Wang C-C. Translational Efficiency of a Non-AUG Initiation Codon Is Significantly Affected by Its Sequence Context in Yeast. J Biol Chem. 2008;283(6):3173-80.

24. Wethmar K. The regulatory potential of upstream open reading frames in eukaryotic gene expression. Wiley Interdisciplinary Reviews: RNA. 2014;5(6):765-8.

25. Llácer JL, Hussain T, Marler L, Aitken CE, Thakur A, Lorsch JR, et al. Conformational Differences between Open and Closed States of the Eukaryotic Translation Initiation Complex. Mol Cell. 2015;59(3):399-412.

26. Hinnebusch AG. Structural Insights into the Mechanism of Scanning and Start Codon Recognition in Eukaryotic Translation Initiation. Trends Biochem Sciences. 2017;42(8):589-611.

27. Llácer JL, Hussain T, Saini AK, Nanda JS, Kaur S, Gordiyenko Y, et al. Translational initiation factor elF5 replaces elF1 on the $40 S$ ribosomal subunit to promote start-codon recognition. eLife. 2018;7:e39273.

28. Janbon G. Introns in Cryptococcus. Memorias do Instituto Oswaldo Cruz. 2018;113(7):e170519-e.

29. Goebels C, Thonn A, Gonzalez-Hilarion S, Rolland O, Moyrand F, Beilharz TH, et al. Introns regulate gene expression in Cryptococcus neoformans in a Pab2 $p$ dependent pathway. PLoS Genet. 2013;9:e1003686.

30. Dumesic PA, Natarajan P, Chen C, Drinnenberg IA, Schiller BJ, Thompson JD, et al. Stalled spliceosomes are a signal for RNAi-mediated genome defense. Cell. 2013;152:957-68.

31. Bonnet A, Grosso AR, Elkaoutari A, Coleno E, Presle A, Sridhara SC, et al. Introns Protect Eukaryotic Genomes from Transcription-Associated Genetic Instability. Mol Cell. 2017;67(4):60821.e6.

32. Janbon G, Ormerod KL, Paulet D, Byrnes III EJ, Chatterjee G, Yadav V, et al. Analysis of the genome and transcriptome of Cryptococcus neoformans var. grubii reveals complex RNA expression and microevolution leading to virulence attenuation. PLoS Genet. 2014;10:e1004261.

33. Gonzalez-Hilarion S, Paulet D, Lee K-T, Hon C-C, Lechat P, Mogensen E, et al. Intron retentiondependent gene regulation in Cryptococcus neoformans. Scientific Reports. 2016;6:32252.

34. Malabat C, Feuerbach F, Ma L, Saveanu C, Jacquier A. Quality control of transcription start site selection by nonsense-mediated-mRNA decay. Elife. 2015;4:e06722.

35. Li H, Hou J, Bai L, Hu C, Tong P, Kang Y, et al. Genome-wide analysis of core promoter structures in Schizosaccharomyces pombe with DeepCAGE. RNA Biology. 2015;12(5):525-37.

36. Ingolia NT, Ghaemmaghami S, Newman JRS, Weissman JS. Genome-Wide Analysis in Vivo of Translation with Nucleotide Resolution Using Ribosome Profiling. Science. 2009;324(5924):218.

37. Hinnebusch AG. TRANSLATIONAL REGULATION OF GCN4 AND THE GENERAL AMINO ACID CONTROL OF YEAST. Ann Rev Microbiol. 2005;59(1):407-50.

38. Duncan CDS, Rodríguez-López M, Ruis P, Bähler J, Mata J. General amino acid control in fission yeast is regulated by a nonconserved transcription factor, with functions analogous to Gcn4/Atf4. Proceedings of the National Academy of Sciences. 2018;115(8):E1829.

39. Madi L, McBride SA, Bailey LA, Ebbole DJ. rco-3, a gene involved in glucose transport and conidiation in Neurospora crassa. Genetics. 1997;146(2):499-508.

40. Wiese A, Elzinga N, Wobbes B, Smeekens S. Sucrose-induced translational repression of plant bZIP-type transcription factors. Biochemical Society Transactions. 2005;33(1):272.

41. Kervestin S, Jacobson A. NMD: a multifaceted response to premature translational termination. Nat Rev Mol Cell Biol. 2012;13:703-12.

42. Hood HM, Spevak CC, Sachs MS. Evolutionary changes in the fungal carbamoyl-phosphate synthetase small subunit gene and its associated upstream open reading frame. Fungal Genet Biol. 2007;44(2):93-104.

43. Danpure CJ. How can the products of a single gene be localized to more than one intracellular compartment? Trends in Cell Biology. 1995;5(6):230-8. 
44. Mireau H, Lancelin D, Small ID. The same Arabidopsis gene encodes both cytosolic and mitochondrial alanyl-tRNA synthetases. The Plant cell. 1996;8(6):1027-39.

45. Mudge SJ, Williams JH, Eyre HJ, Sutherland GR, Cowan PJ, Power DA. Complex organisation of the 5? ? -end of the human glycine tRNA synthetase gene. Gene. 1998;209(1):45-50.

46. Natsoulis G, Hilger F, Fink GR. The HTS1 gene encodes both the cytoplasmic and mitochondrial histidine tRNA synthetases of $S$. cerevisiae. Cell. 1986;46(2):235-43.

47. Kriventseva EV, Kuznetsov D, Tegenfeldt F, Manni M, Dias R, Simão FA, et al. OrthoDB v10: sampling the diversity of animal, plant, fungal, protist, bacterial and viral genomes for evolutionary and functional annotations of orthologs. Nucleic acids research. 2019;47(D1):D807-D11.

48. Muruganujan A, Mi H, Thomas PD. PANTHER in 2013: modeling the evolution of gene function, and other gene attributes, in the context of phylogenetic trees. Nucleic Acids Research. 2012;41(D1):D377-D86.

49. Datt $M$, Sharma A. Novel and unique domains in aminoacyl-tRNA synthetases from human fungal pathogens Aspergillus niger, Candida albicans and Cryptococcus neoformans. BMC Genomics. 2014;15(1):1069.

50. Duchêne A-M, Pujol C, Maréchal-Drouard L. Import of tRNAs and aminoacyl-tRNA synthetases into mitochondria. Curr Genet. 2009;55(1):1-18.

51. Muruganujan A, Ebert $\mathrm{D}, \mathrm{Mi} H$, Thomas PD, Huang X. PANTHER version 14: more genomes, a new PANTHER GO-slim and improvements in enrichment analysis tools. Nucleic Acids Res. 2018;47(D1):D419-D26.

52. Frechin M, Duchêne A-M, Becker HD. Translating organellar glutamine codons : A case by case scenario? RNA Biology. 2009;6(1):31-4.

53. Chang C-P, Tseng $Y-K$, Ko $C-Y$, Wang C-C. Alanyl-tRNA synthetase genes of Vanderwaltozyma polyspora arose from duplication of a dual-functional predecessor of mitochondrial origin. Nucleic Acids Res. 2012;40(1):314-22.

54. Geslain R, Martin F, Delagoutte B, Cavarelli J, Gangloff J, Eriani G. In vivo selection of lethal mutations reveals two functional domains in arginyl-tRNA synthetase. RNA (New York, NY). 2000;6(3):434-48.

55. Merz S, Westermann B. Genome-wide deletion mutant analysis reveals genes required for respiratory growth, mitochondrial genome maintenance and mitochondrial protein synthesis in Saccharomyces cerevisiae. Genome Biol. 2009;10(9):R95.

56. Sickmann A, Reinders J, Wagner Y, Joppich C, Zahedi R, Meyer HE, et al. The proteome of Saccharomyces cerevisiae mitochondria. Proceedings of the National Academy of Sciences of the United States of America. 2003;100(23):13207-12.

57. Chen S-J, Wu Y-H, Huang H-Y, Wang C-C. Saccharomyces cerevisiae Possesses a StressInducible Glycyl-tRNA Synthetase Gene. PLOS ONE. 2012;7(3):e33363.

58. Chiu W-C, Chang C-P, Wen W-L, Wang S-W, Wang C-C. Schizosaccharomyces pombe Possesses Two Paralogous Valyl-tRNA Synthetase Genes of Mitochondrial Origin. Mol Biol Evol. 2010;27(6):1415-24.

59. Ivanov IP, Loughran G, Sachs MS, Atkins JF. Initiation context modulates autoregulation of eukaryotic translation initiation factor 1 (elF1). Proceedings of the National Academy of Sciences of the United States of America. 2010;107(42):18056-60.

60. Loughran G, Sachs MS, Atkins JF, Ivanov IP. Stringency of start codon selection modulates autoregulation of translation initiation factor elF5. Nucleic Acids Res. 2012;40(7):2898-906.

61. Martin-Marcos P, Cheung Y-N, Hinnebusch AG. Functional elements in initiation factors $1,1 \mathrm{~A}$, and $2 \beta$ discriminate against poor AUG context and non-AUG start codons. Mol Cell Biol. 2011;31(23):4814-31.

62. Hussain T, Llácer JL, Fernández IS, Munoz A, Martin-Marcos P, Savva CG, et al. Structural changes enable start codon recognition by the eukaryotic translation initiation complex. Cell. 2014;159(3):597-607. 
63. Thakur A, Hinnebusch AG. elF1 Loop 2 interactions with Met-tRNA(i) control the accuracy of start codon selection by the scanning preinitiation complex. Proceedings of the National Academy of Sciences of the United States of America. 2018;115(18):E4159-E68.

64. Olsen DS, Savner EM, Mathew A, Zhang F, Krishnamoorthy T, Phan L, et al. Domains of elF1A that mediate binding to elF2, elF3 and elF5B and promote ternary complex recruitment in vivo. The EMBO journal. 2003;22(2):193-204.

65. Luna RE, Arthanari H, Hiraishi $\mathrm{H}$, Akabayov B, Tang L, Cox C, et al. The interaction between eukaryotic initiation factor $1 \mathrm{~A}$ and elF5 retains elF1 within scanning preinitiation complexes. Biochemistry. 2013;52(52):9510-8.

66. Fekete CA, Applefield DJ, Blakely SA, Shirokikh N, Pestova T, Lorsch JR, et al. The elF1A Cterminal domain promotes initiation complex assembly, scanning and AUG selection in vivo. The EMBO journal. 2005;24(20):3588-601.

67. Slusher LB, Gillman EC, Martin NC, Hopper AK. mRNA leader length and initiation codon context determine alternative AUG selection for the yeast gene MOD5. Proceedings of the National Academy of Sciences. 1991;88(21):9789.

68. Calvo SE, Pagliarini DJ, Mootha VK. Upstream open reading frames cause widespread reduction of protein expression and are polymorphic among humans. Proceedings of the National Academy of Sciences of the United States of America. 2009;106(18):7507-12.

69. Cheng Z, Otto GM, Powers EN, Keskin A, Mertins P, Carr SA, et al. Pervasive, Coordinated Protein-Level Changes Driven by Transcript Isoform Switching during Meiosis. Cell. 2018;172(5):91023.e16.

70. Van Dalfsen KM, Hodapp S, Keskin A, Otto GM, Berdan CA, Higdon A, et al. Global Proteome Remodeling during ER Stress Involves Hac1-Driven Expression of Long Undecoded Transcript Isoforms. Developmental Cell. 2018;46(2):219-35.e8.

71. Monteuu is G, Miścicka A, Świrski M, Zenad L, Niemitalo O, Wrobel L, et al. Non-canonical translation initiation in yeast generates a cryptic pool of mitochondrial proteins. Nucleic Acids Res. 2019:in press.

72. Brar GA. Beyond the Triplet Code: Context Cues Transform Translation. Cell. 2016;167(7):1681-92.

73. Feeney KA, Hansen LL, Putker M, Olivares-Yañez C, Day J, Eades L, et al. Daily magnesium fluxes regulate cellular timekeeping and energy balance. Nature. 2016;532(7599):375-9.

74. Tsuboi T, Viana MP, Xu F, Yu J, Chanchani R, Arceo XG, et al. Mitochondrial volume fraction controls translation of nuclear-encoded mitochondrial proteins. bioRxiv. 2019:529289.

75. Nakagawa S, Niimura Y, Gojobori T, Tanaka H, Miura K-i. Diversity of preferred nucleotide sequences around the translation initiation codon in eukaryote genomes. Nucleic acids research. 2008;36(3):861-71.

76. Shah M, Su D, Scheliga JS, Pluskal T, Boronat S, Motamedchaboki K, et al. A TranscriptSpecific elF3 Complex Mediates Global Translational Control of Energy Metabolism. Cell reports. 2016;16(7):1891-902.

77. Fields SD, Conrad MN, Clarke M. The S. cerevisiae CLU1 and D. discoideum cluA genes are functional homologues that influence mitochondrial morphology and distribution. Journal of Cell Science. 1998;111(12):1717.

78. Gao J, Schatton D, Martinelli P, Hansen H, Pla-Martin D, Barth E, et al. CLUH regulates mitochondrial biogenesis by binding mRNAs of nuclear-encoded mitochondrial proteins. The Journal of Cell Biology. 2014;207(2):213.

79. Schatton D, Pla-Martin D, Marx M-C, Hansen H, Mourier A, Nemazanyy I, et al. CLUH regulates mitochondrial metabolism by controlling translation and decay of target mRNAs. The Journal of cell biology. 2017;216(3):675-93.

80. Smith MD, Gu Y, Querol-Audí J, Vogan JM, Nitido A, Cate JHD. Human-Like Eukaryotic Translation Initiation Factor 3 from Neurospora crassa. PLOS ONE. 2013;8(11):e78715.

81. Madhani HD. The frustrated gene: origins of eukaryotic gene expression. Cell. 2013;155(4):744-9. 
82. Winston F, Dollard C, Ricupero-Hovasse SL. Construction of a set of convenient saccharomyces cerevisiae strains that are isogenic to S288C. Yeast. 1995;11(1):53-5.

83. Kim D, Pertea G, Trapnell C, Pimentel H, Kelley R, Salzberg SL. TopHat2: accurate alignment of transcriptomes in the presence of insertions, deletions and gene fusions. Genome Biol. 2013;14(4):R36-R.

84. Martin M. Cutadapt removes adapter sequences from high-throughput sequencing reads. EMBnetjournal; Vol 17, No 1: Next Generation Sequencing Data Analysis. 2011.

85. Dunn JG, Foo CK, Belletier NG, Gavis ER, Weissman JS. Ribosome profiling reveals pervasive and regulated stop codon readthrough in Drosophila melanogaster. eLife. 2013;2:e01179.

86. Carja O, Xing T, Wallace EWJ, Plotkin JB, Shah P. riboviz: analysis and visualization of ribosome profiling datasets. BMC bioinformatics. 2017;18(1):461-.

87. Pertea M, Kim D, Pertea GM, Leek JT, Salzberg SL. Transcript-level expression analysis of RNAseq experiments with HISAT, StringTie and Ballgown. Nature Protocols. 2016;11:1650.

88. Li H, Handsaker B, Wysoker A, Fennell T, Ruan J, Homer N, et al. The Sequence Alignment/Map format and SAMtools. Bioinformatics (Oxford, England). 2009;25(16):2078-9.

89. Quinlan AR, Hall IM. BEDTools: a flexible suite of utilities for comparing genomic features. Bioinformatics (Oxford, England). 2010;26(6):841-2.

90. Team RC. R: A language and environment for statistical computing. R Foundation for Statistical Computing, Vienna, Austria. Available online at https://www.R-project.org/. 2018.

91. Wickham H, editor. ggplot2: Elegant Graphics for Data Analysis: Springer-Verlag New York; 2016.

92. Wickham H, François R, Henry L, Müller K. dplyr: A Grammar of Data Manipulation. R package version 0.7.8. https://CRAN.R-project.org/package=dplyr. 2018.

93. Wilke CO. cowplot: Streamlined Plot Theme and Plot Annotations for 'ggplot2'. R package version 0.9.3. https://CRAN.R-project.org/package=cowplot. 2018.

94. Wagih O. ggseqlogo: A 'ggplot2' Extension for Drawing Publication-Ready Sequence Logos. $R$ package version 0.1. https://CRAN.R-project.org/package=ggseqlogo. 2017.

95. Love MI, Huber W, Anders S. Moderated estimation of fold change and dispersion for RNAseq data with DESeq2. Genome Biol. 2014;15:550.

96. Edgar RC. MUSCLE: multiple sequence alignment with high accuracy and high throughput. Nucleic Acids Res. 2004;32(5):1792-7.

97. Wilm A, Higgins DG, Valentin F, Blackshields G, McWilliam H, Wallace IM, et al. Clustal W and Clustal $X$ version 2.0. Bioinformatics. 2007;23(21):2947-8.

98. Yu C-H, Dang Y, Zhou Z, Wu C, Zhao F, Sachs MS, et al. Codon Usage Influences the Local Rate of Translation Elongation to Regulate Co-translational Protein Folding. Mol Cell. 2015;59(5):744-54.

99. Kersey PJ, Allen JE, Allot A, Barba M, Boddu S, Bolt BJ, et al. Ensembl Genomes 2018: an integrated omics infrastructure for non-vertebrate species. Nucleic Acids Res. 2018;46(D1):D802-D8. 100. Duncan CDS, Mata J. Effects of cycloheximide on the interpretation of ribosome profiling experiments in Schizosaccharomyces pombe. Scientific Reports. 2017;7(1):10331.

101. Muzzey D, Sherlock G, Weissman JS. Extensive and coordinated control of allele-specific expression by both transcription and translation in Candida albicans. Genome Res. 2014;24(6):96373.

102. Skrzypek MS, Binkley J, Binkley G, Miyasato SR, Simison M, Sherlock G. The Candida Genome Database (CGD): incorporation of Assembly 22, systematic identifiers and visualization of high throughput sequencing data. Nucleic Acids Res. 2017;45(D1):D592-D6.

103. Gerashchenko MV, Gladyshev VN. Translation inhibitors cause abnormalities in ribosome profiling experiments. Nucleic Acids Res. 2014;42(17):e134-e.

104. Csárdi G, Franks A, Choi DS, Airoldi EM, Drummond DA. Accounting for Experimental Noise Reveals That mRNA Levels, Amplified by Post-Transcriptional Processes, Largely Determine SteadyState Protein Levels in Yeast. PLoS Genet. 2015;11(5):e1005206. 
105. Weinberg DE, Shah P, Eichhorn SW, Hussmann JA, Plotkin JB, Bartel DP. Improved RibosomeFootprint and mRNA Measurements Provide Insights into Dynamics and Regulation of Yeast Translation. Cell reports. 2016;14(7):1787-99.

106. Cherry JM, Hong EL, Amundsen C, Balakrishnan R, Binkley G, Chan ET, et al. Saccharomyces Genome Database: the genomics resource of budding yeast. Nucleic Acids Res. 2012;40(Database issue):D700-D5.

107. Basenko YE, Pulman AJ, Shanmugasundram A, Harb SO, Crouch K, Starns D, et al. FungiDB: An Integrated Bioinformatic Resource for Fungi and Oomycetes. Journal of Fungi. 2018;4(1).

108. Ban N, Beckmann R, Cate JHD, Dinman JD, Dragon F, Ellis SR, et al. A new system for naming ribosomal proteins. Current opinion in structural biology. 2014;24:165-9. 

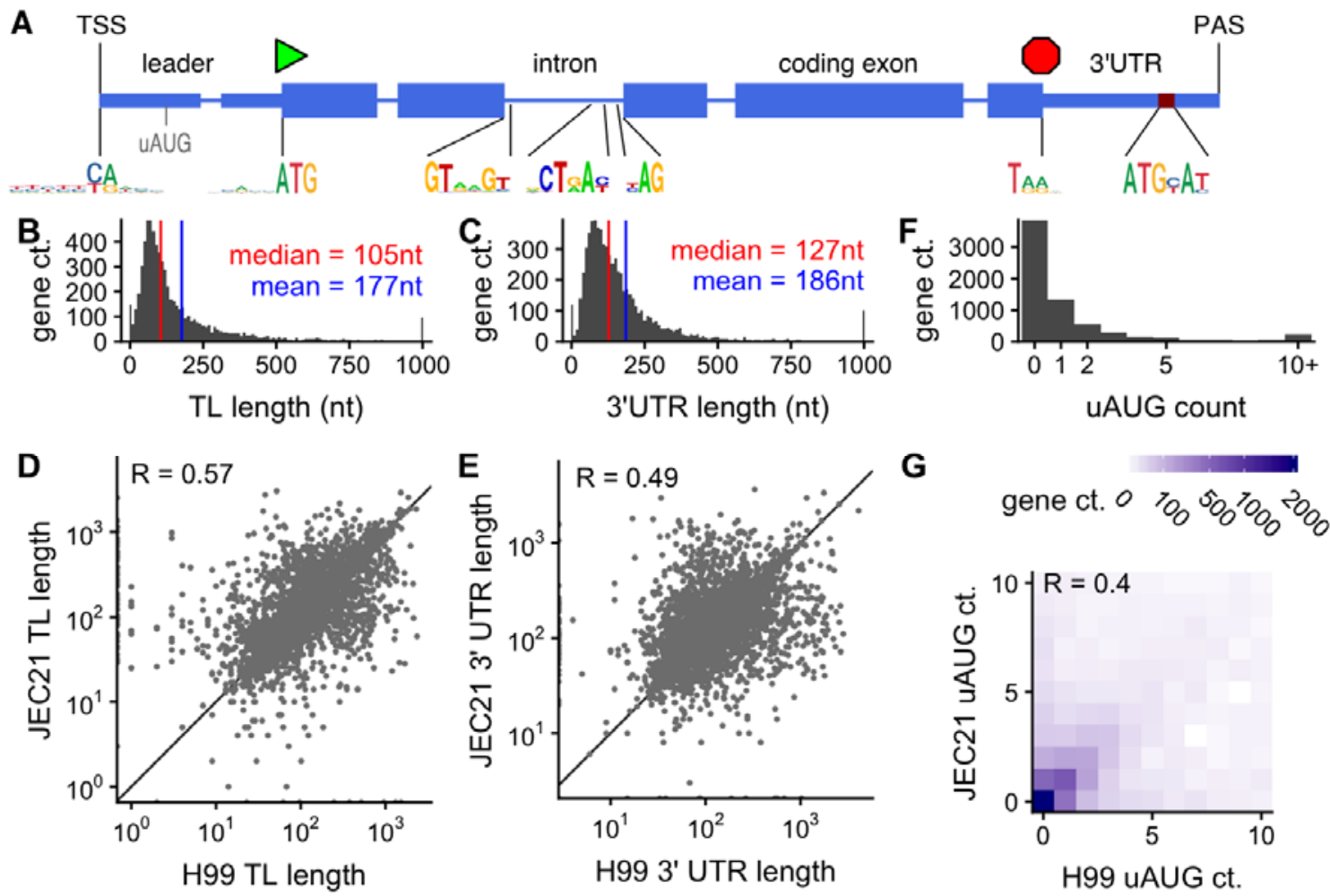

Figure 1: Mapping the coding transcriptome of Cryptococcus neoformans. A,

Representation of a stereotypical gene of $C$. neoformans $\mathrm{H} 99$, showing the sequence logos for the transcription start site (TSS), AUG start codon, intron splicing, stop codon, and polyadenylation site (PAS). B, Distribution of transcript leader (TL) lengths over $C$. neoformans genes, for yeast cells growing exponentially in YPD at $30^{\circ} \mathrm{C}$. B, Distribution of $3^{\prime}$ untranslated region ( $3^{\prime} U T R$ ) lengths over $C$. neoformans genes. C,D : Comparisons of TL and 3'UTR lengths between orthologous genes in C. neoformans $\mathrm{H} 99$ and $\mathrm{C}$. deneoformans JEC21 growing exponentially in YPD at $30^{\circ} \mathrm{C}$. F, Distribution of upstream AUG (UAUG) counts over $C$. neoformans genes, and $\mathrm{G}$, comparison of UAUG counts with $C$. deneoformans. 


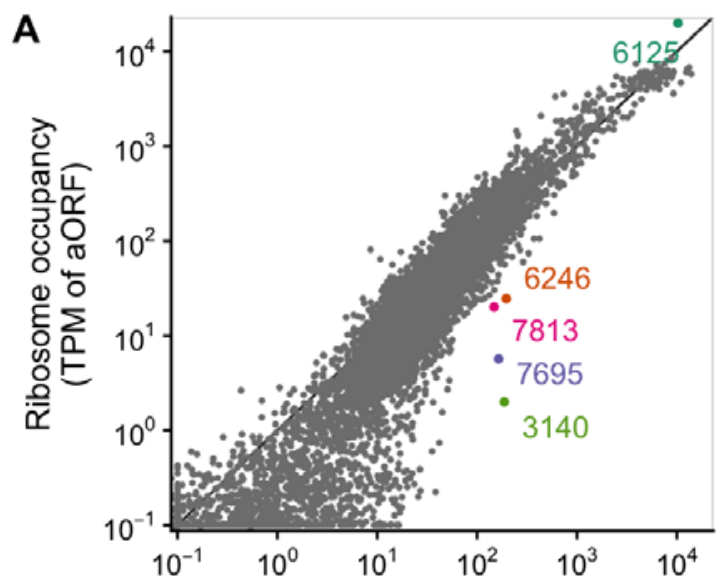

RNA abundance (TPM of aORF)

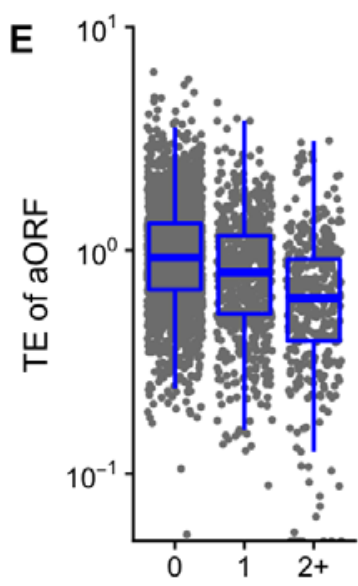

uAUG count

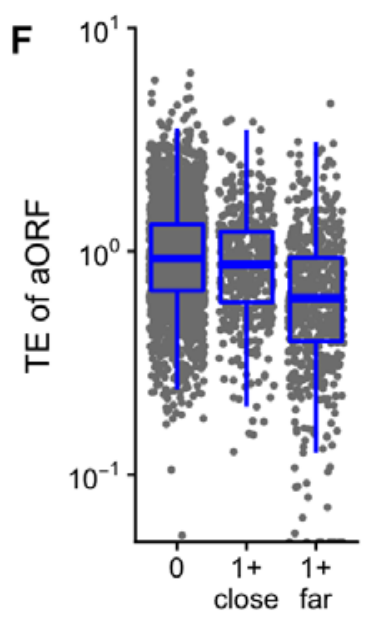

uAUG count

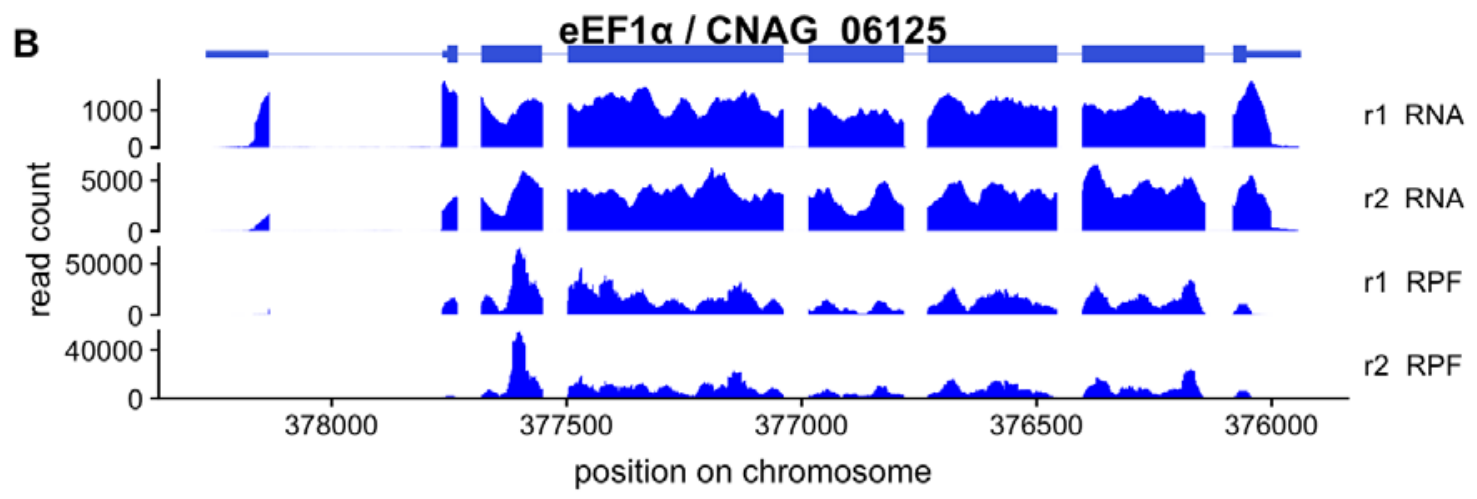

C

CNAG 06246

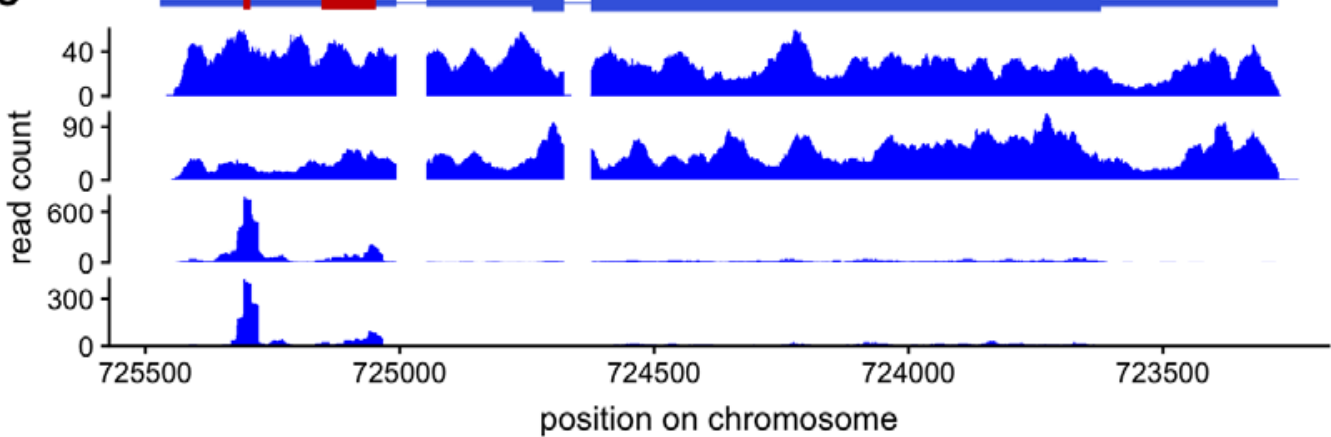

r1 RNA

r2 RNA

r1 RPF

r2 RPF

D

CNAG 03140

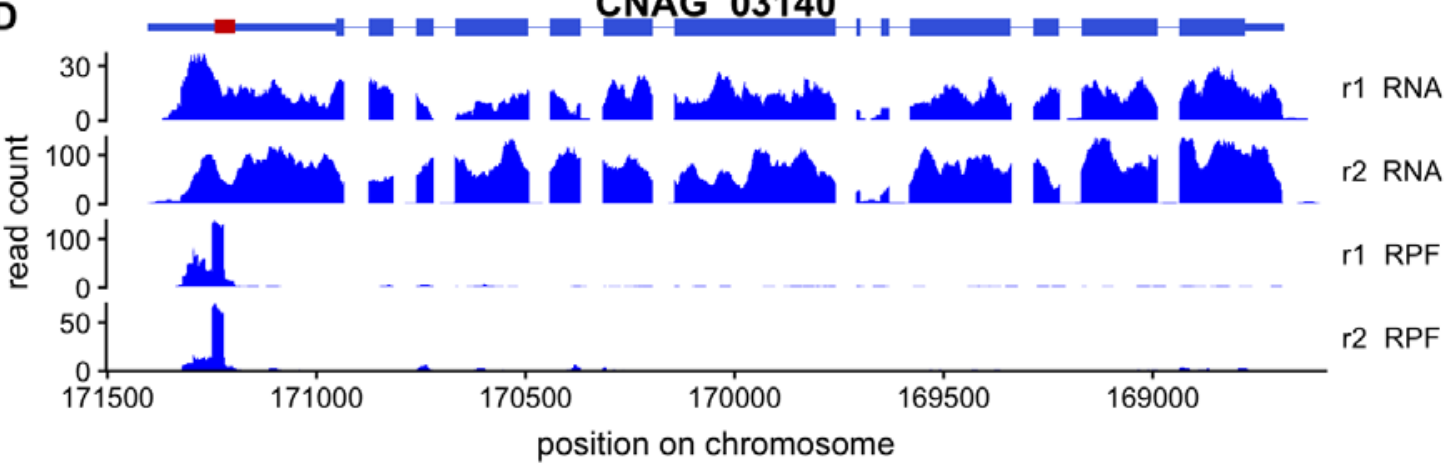


Figure 2: Upstream AUGs repress translation in C. neoformans. A, translation regulation of annotated ORFs (aORFs) in C. neoformans $\mathrm{H} 99$ growing exponentially in YPD at $30^{\circ} \mathrm{C}$ (equivalent data for $C$. deneoformans shown in Fig S2.1). Ribosome occupancy is plotted against the RNA abundance, both calculated in transcripts per million (TPM) on the aORF. Select genes discussed in the text are highlighted in colour. B, UAUGs are associated with lower translation efficiency (TE) of annotated ORFs, measured as the ratio of ribosome occupancy to RNA-seq reads. C, only uAUGs far from the transcription start site are associated with low TE. A gene is in the " $1+$ far" category if it has at least one UAUG more than 20nt from the TSS, "1+ close" if all UAUGs are within 20nt of the TSS. D-F, Examples of ribosome occupancy profiles along select RNAs highlighted in 2A (others are shown in Fig S2.2). D, Translation elongation factor eEF2/CNAG_06125 has high ribosome occupancy in the annotated ORF. Translationally repressed mRNAs CNAG_06246 (E) and CNAG_03140 (F) have high ribosome occupancy in uORFs in the transcript leader (red), and low ribosome occupancy in the aORF. Only the first of 5 uORFs in CNAG_03140 is shown. Homologous genes in $\mathrm{C}$. deneoformans have similar structure and regulation (Fig S2.1). 

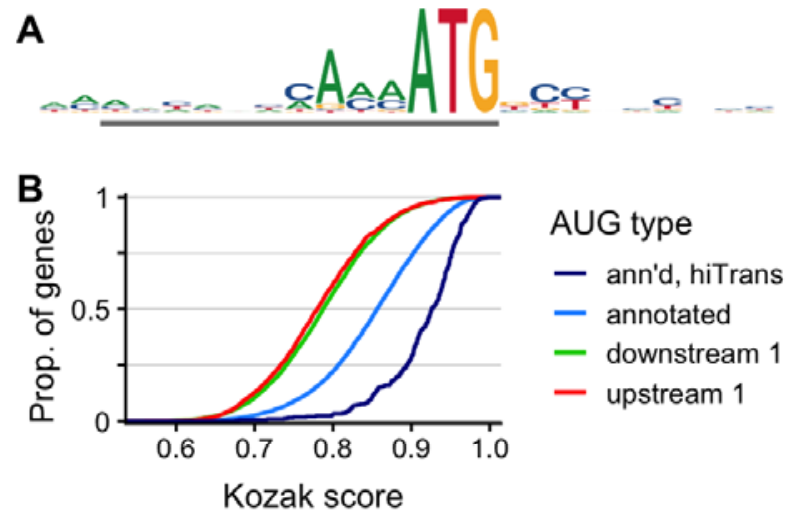

D
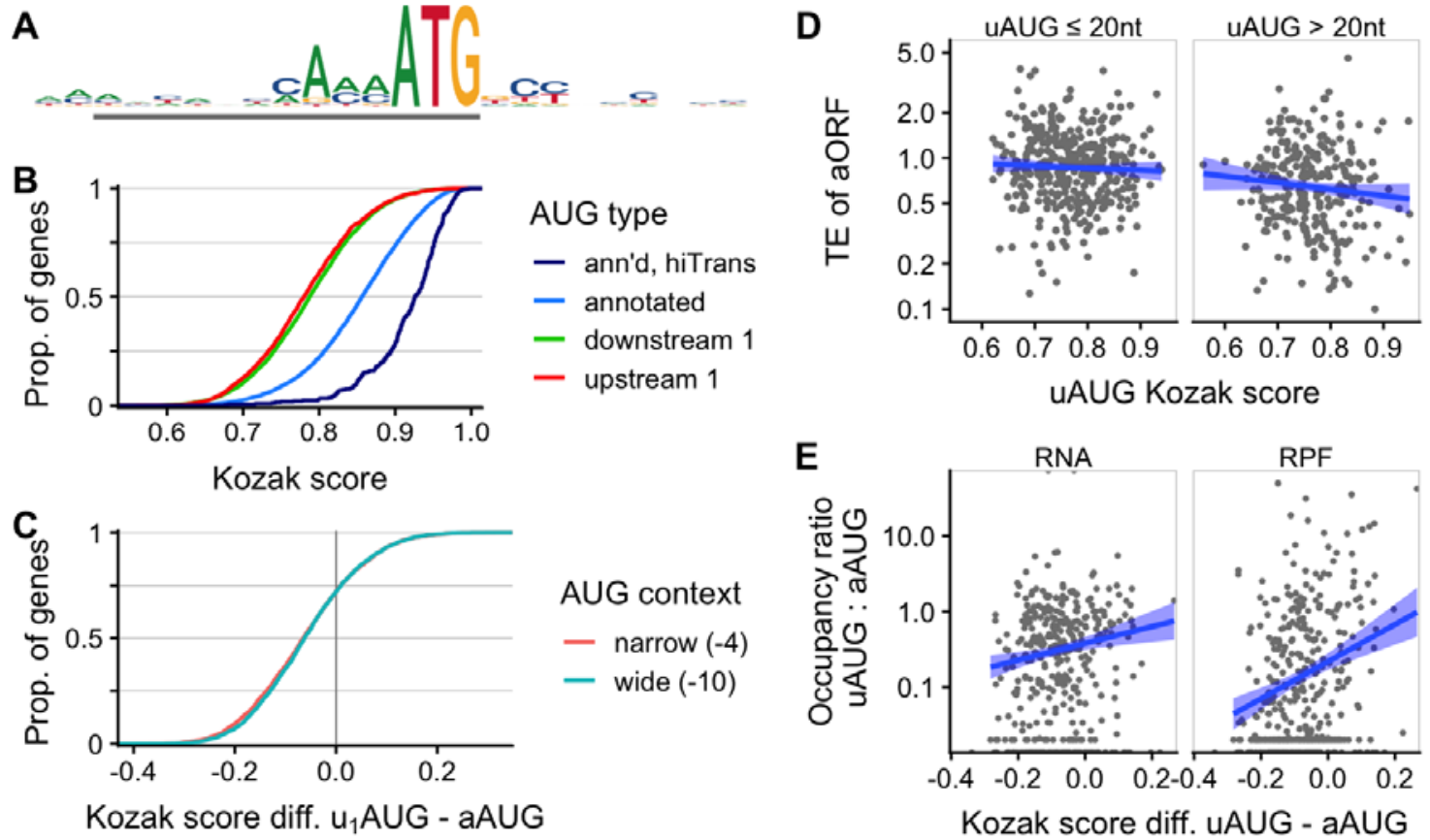

Figure 3: An AUG sequence context is associated with translation in C. neoformans. A, Kozak-like sequence context of AUGs, from -12 to +12 , for highest-translated $5 \%$ of genes (hiTrans). This sequence context is used to create "Kozak scores" of other AUG sequences by their similarity to the consensus from -10 onwards. B, Cumulative density plot of Kozak scores from various categories of AUG, showing that high scores are associated with annotated AUGs of highly translated genes (hiTrans), somewhat with annotated AUGs, and not with the most 5' downstream AUG (downstream 1) or 5' most upstream AUG (upstream 1) in a transcript. C, Cumulative density plot of differences in scores between most $5^{\prime}$ upstream ( $u_{1} A U G$ ) and annotated $A U G$, showing that for $75 \%$ of genes the upstream AUG score is less than the annotated AUG, whether we take a wide (-10:AUG) or a narrow (4:AUG) window to calculate the score. D, High upstream AUG score is weakly associated with translation repression of the annotated ORF, if the UAUG is further than 20nt from the TSS. $E$, The relative occupancy of ribosomes (RPF) at the upstream AUG and annotated AUG depends on the difference in scores, even when compared to RNA-seq reads; linear model trend fit shown (blue). Panels D and E show data only for genes in the top $50 \%$ by RNA abundance, and with only a single upstream AUG. 


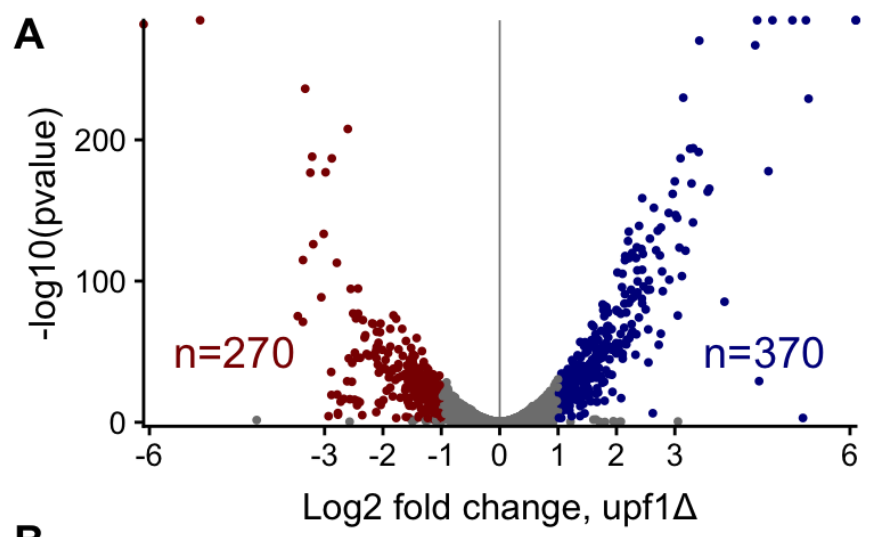

B

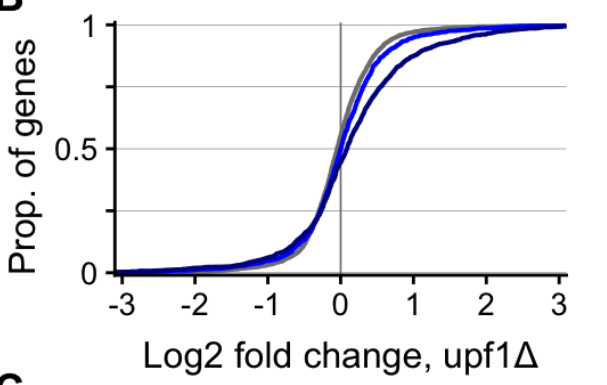

uAUG count

$-0$

$-1$

$-2+$

C

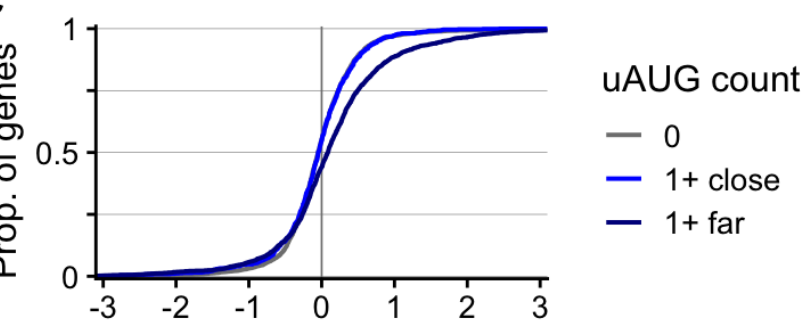

\section{D}

Log2 fold change, upf1 $\Delta$

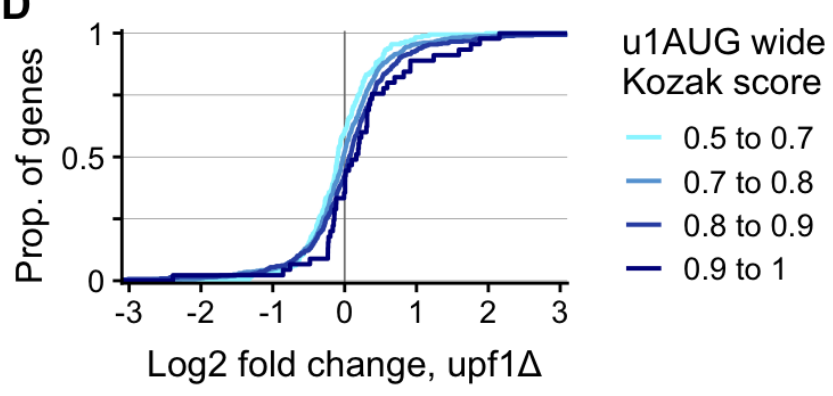

Figure 4: Nonsense-mediated decay (NMD) acts on upstream-ORF-containing mRNAs in $C$. deneoformans. A, Differential expression results from RNA-Seq in C. deneoformans JEC21, comparing expression in wild-type cells with a mutant deleted for NMD factor UPF1/CNC02960, and using DeSeq2 to identify genes upregulated in the upf1 $1 \triangle$ mutant. B, UORF containing genes are enriched for NMD-sensitivity. C, uORF-containing genes are enriched for NMD-sensitivity only when the UAUG is more than 20nts from the TSS (1+far), but not when the uAUG is less than 20nts (1+close). D, Start codon sequence context affects NMD sensitivity of genes containing a single upstream AUG: UORFs starting with higher Kozak-score uAUG are more likely to increase in abundance in the upf1 $\triangle$ mutant. 

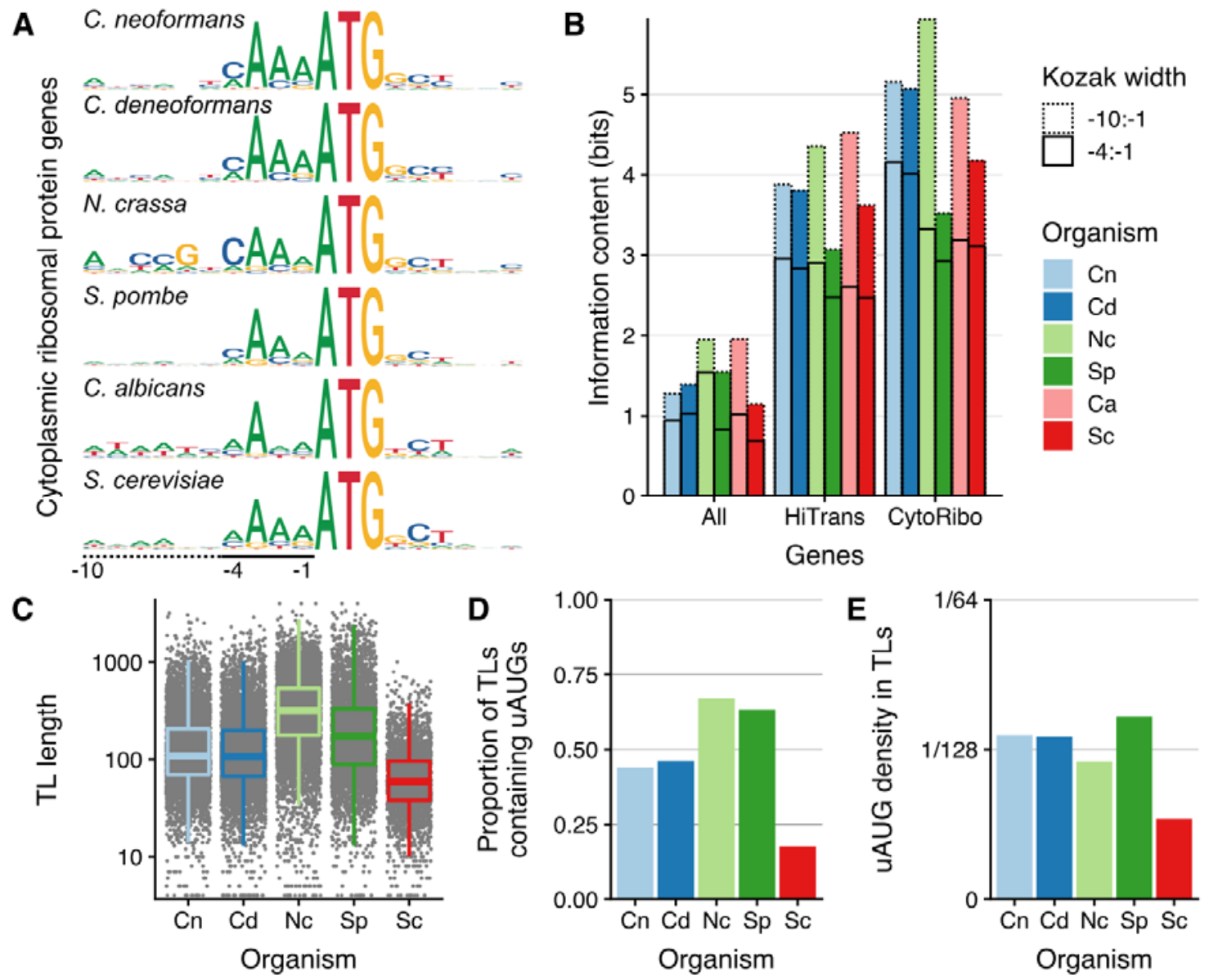

Figure 5: Sequences specifying start codon selection are quantitatively different in

different fungi. A, Kozak consensus sequence logo for annotated start codons of cytoplasmic ribosomal protein genes from 6 fungal species. The height of each letter represents the Shannon information content in bits, so that the anchor ATG sequence has height 2 bits. B, Information content at annotated start codons in bits per base (i.e. summed height of stacked letters in sequence logo) for 3 groups of genes, in the 6 fungi from panel A. Solid line indicates information from -1 to -4 of ATG, and dotted line additionally to -10 (see bottom of panel A). Gene groups are all annotated ORFs, highly translated ORFs (HiTrans) and cytoplasmic ribosomal proteins (CytoRibo, as panel A). HiTrans used the highest-translated $5 \%$ of genes, or the highest 400 genes for fungi with more than 8000 annotated genes ( $C$. albicans and N. crassa; see methods). C-E, For 5 fungi for which transcript leader (TL) annotations were available, TL length (C), proportion of annotated TL containing an upstream AUG (D), and proportions of AUGs per nucleotide in the TL (E; a uniform random model would have density $1 / 64$ ). 

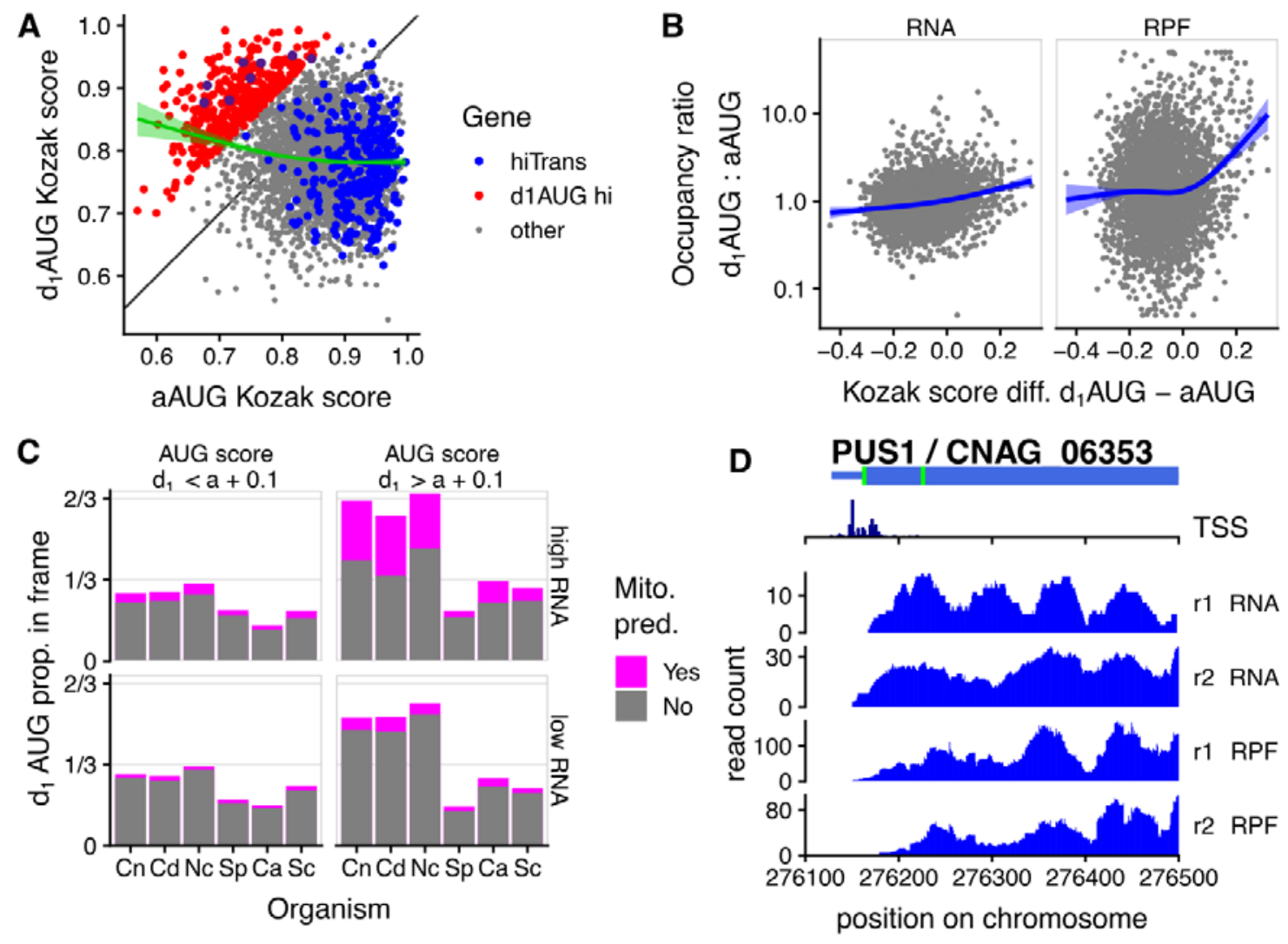

Figure 6: High-scoring downstream AUGs specify alternative $\mathbf{N}$-terminal isoforms in $\mathbf{C}$. neoformans. A, Most genes with reasonable RNA abundance (top $50 \%$ by RNA abundance shown), especially very highly-translated genes (blue, top 5\%), have lower Kozak score at the 1st downstream AUG than at the annotated AUG. However there are exceptions (red, $d_{1} A U G$ hi: $d_{1} A U G$ score $>$ annotated AUG score +0.1 ), and there is a trend for genes with low aAUG score to have a higher $d_{1}$ AUG score (green, generalized additive model fit). B, Higher $d_{1} A U G$ score than aAUG score drives higher ribosome protected fragment (RPF) occupancy at the $d_{1} A U G$ compared to the aAUG, but much smaller differences in RNA-seq density. Blue line indicates generalized additive model fit. C, Downstream AUGs with high Kozak scores $\left(d_{1}\right.$ AUG score > annotated AUG score +0.1$)$ and reasonable RNA abundance (top 50\%) are likely to be in-frame and enriched for $\mathrm{N}$-terminal mitochondrial localization signals in $\mathrm{C}$. neoformans, C. deneoformans, and N. crassa, but not in S. pombe, C. albicans, or S. cerevisiae. D, The pseudouridine synthase CnPus1 is a candidate alternate-localized protein with a low-score aAUG and high-score $d_{1} A U G$, and transcription start sites on both sides of the aAUG. RNA-Seq and RPF reads on the first exon are shown, and the full length of the gene shown in Fig S6.1. 

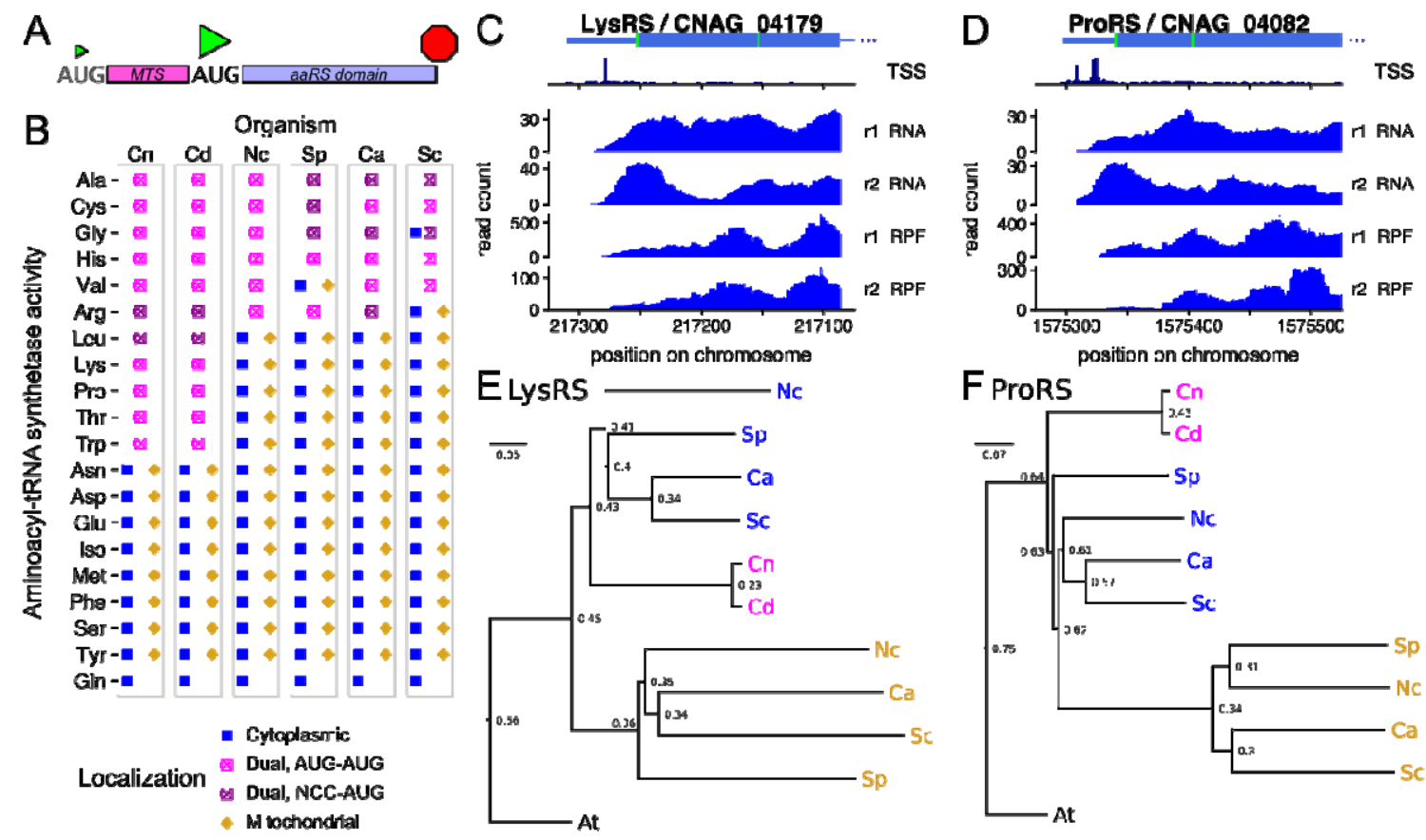

Figure 7: Aminoacyl-tRNA synthetases (aaRSs) are commonly alternatively localized to cytoplasm and mitochondria by use of alternative start codons in fungi. A, Schematic of the structure of a dual-localized aaRS with alternate AUG start codons. B, Predicted localization of all aaRS enzymes in the fungi $C$. neoformans ( $\mathrm{Cn}$ ), C. deneoformans (Cd), N. crassa (NC), $S$. pombe (Sp), C. albicans (Ca), S. cerevisiae (SC). C/D, Transcription start site reads, RNA-seq, and ribosome profiles of 5 '-ends of CnLysRS (C) and CnProRS (D) show that most transcription starts upstream of both AUG start codons (green), and both AUG codons are used for translation initiation. E/F Simplified neighbour-joining phylogenetic trees show that LysRS (E) and ProRS (F) genes were duplicated in ascomycete fungi, and Cryptococcus retained a single dual-localized homolog. Arabidopsis thaliana (At) was used as an out-group. The scale bar represents the number of amino acid substitutions per residue, and the numbers at nodes are the proportion of substitutions between that node and its parent. See table S5, for details of identifiers for genes (GeneID). 


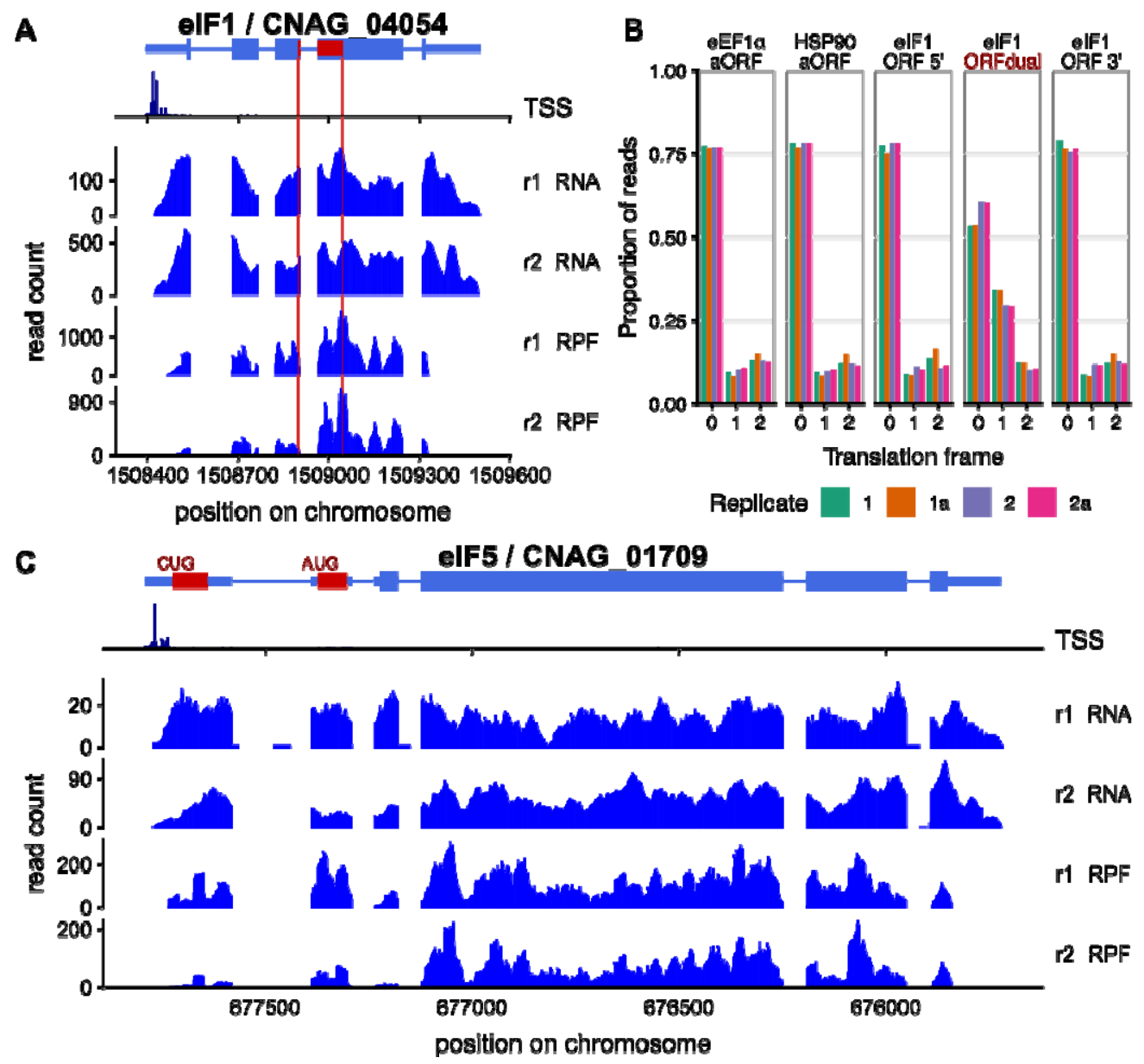

Figure 8. Translation initiation factors elF1 and elF5 are regulated by alternate start codon usage in C. neoformans. A, Reads on CnelF1/CNAG_04054, showing frame +1 "downstream ORF" in dark red, breaking for an intron. B, The downstream ORF of CnelF1 is dual-translated in two frames. Most ribosome profiling read $5^{\prime}$ ends are in a consistent frame, including in control genes eEF1alpha/CNAG_06125 and HSP90/CNAG_06125, and in the 5' and 3' ends of the CnelF1 ORF, but there is $2 x$ enrichment of reads in frame +1 in the dual-decoded ORF. $C$, Reads on CnelF5/CNAG_01709 showing substantial ribosomal occupancy over upstream ORFs. The first upstream ORF shown is translated from a CUG start codon and the second from an AUG codon, and other UORFS potentially initiated from near-cognate codons are not shown. C. deneoformans homologs have the same structure and regulation (Figure S8). 

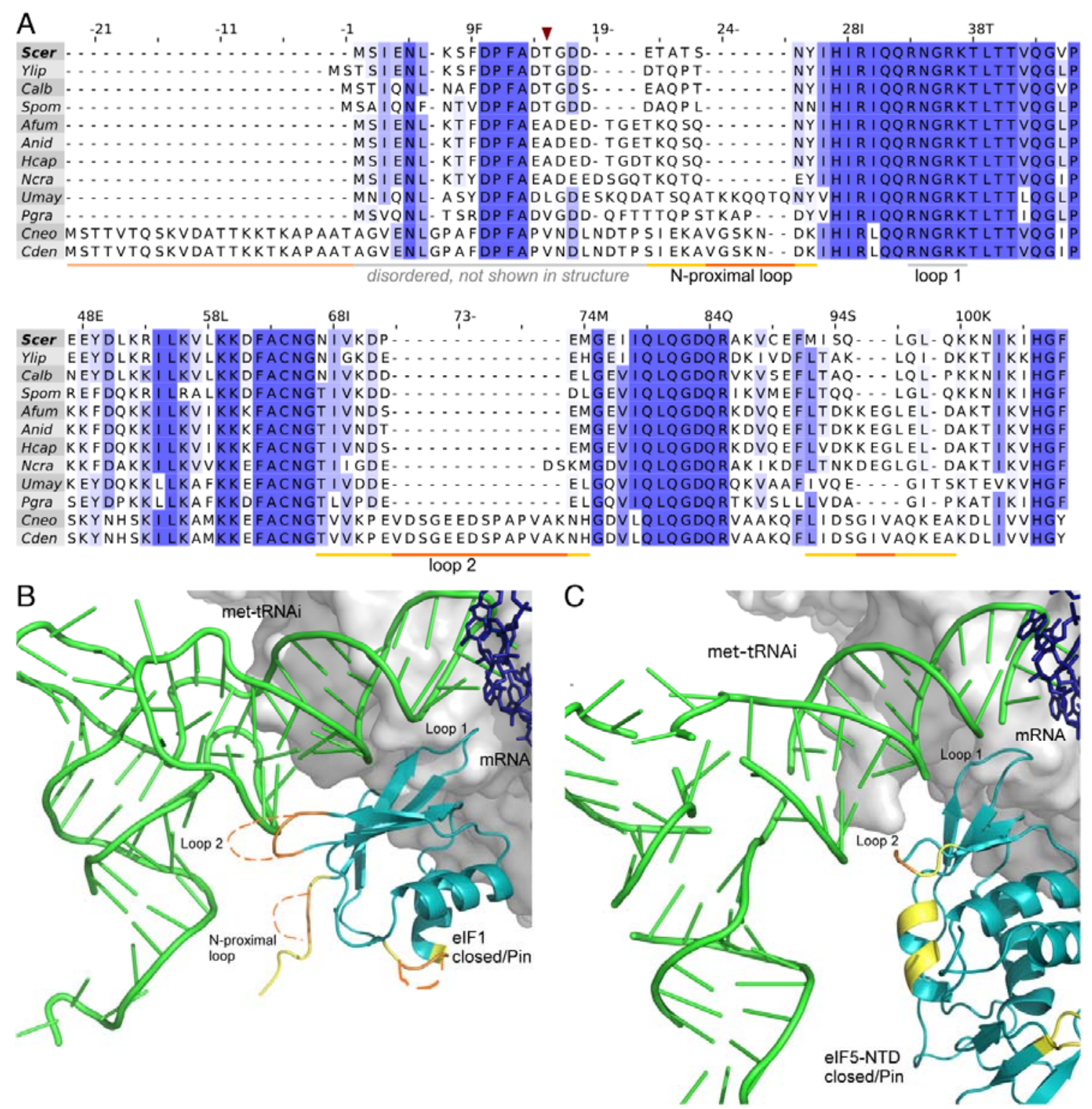

Figure 9. Eukaryotic translation initiation factor 1 is highly variable across fungi. A, Multiple sequence alignment of translation initiation factor elF1 from 12 fungi, numbered as $S$. cerevisiae (Scer, top line). Cryptococcus insertions are indicated in orange, and surrounding variable residues in yellow. The $\mathrm{N}$-terminal extension in Cryptococcus elF1, that is predicted disorded, is shown in pale orange, and T15 residue with dark red arrow. B, Structural predictions of insertions (orange) and non-conserved neighborhoods (yellow) in Cryptococcus elF1 mapped onto the closed pre-initiation complex of S. cerevisiae/K.lactis (PDB:3J81, Hussein 2015). elF1 (teal) and Met-tRNAi (green) in closed conformation, shown with synthetic mRNA sequence (pink), and eIF2 (pale pink) and ribosomal subunit surface (greys) in background. Approximate ribosomal contacts are shown as grey background surface and elF2-alpha subunit is shown as pale pink sticks. B, Structural predictions of variations in Cryptococcus elF5 mapped on to S. cerevisiae PIC (PDB:6FYX, (27)). Multiple sequence alignment of eIF5 is shown in figure S9.1A. 

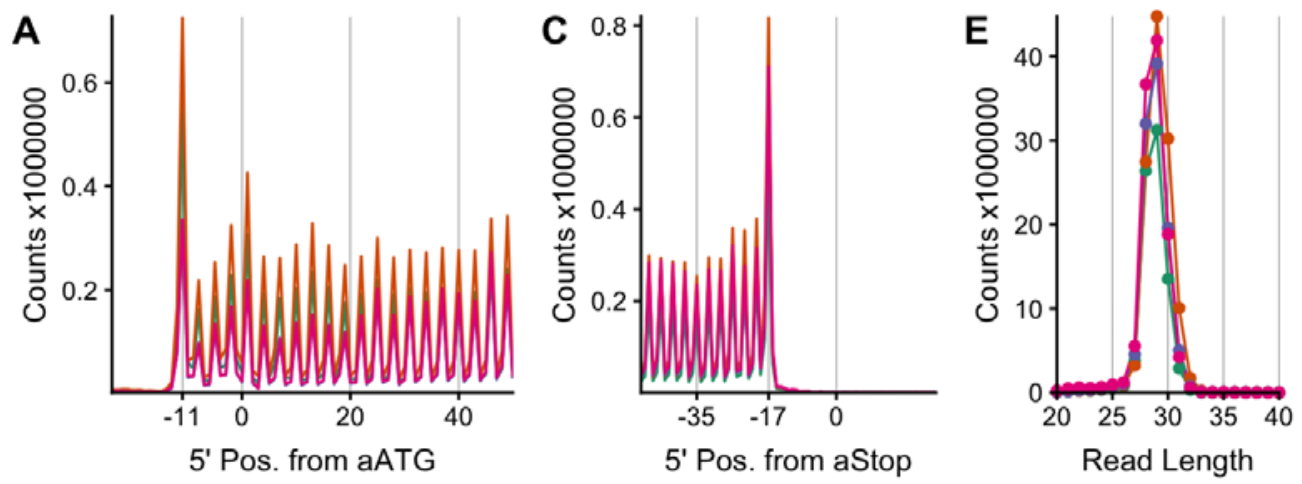

H99

Replicate

$\rightarrow 1$

$\rightarrow 1 \mathrm{a}$

$\rightarrow 2$

$\rightarrow 2 a$
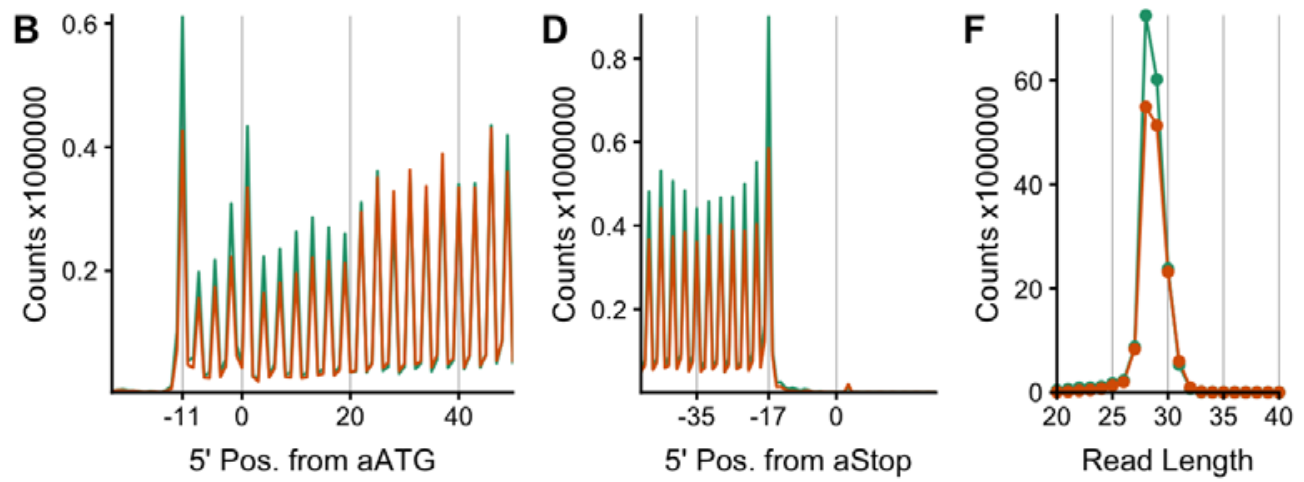

JEC21

Replicate

$\rightarrow 1$

$\rightarrow 2$

Figure S2.1, related to figure 2: Ribosome profiling data passes quality control metrics.

Metagene profiles of mapped 5' ends of ribosome-protected fragment counts at the 5' end $(A, B)$ and 3' end (C,D) of ORFs, showing 3-nucleotide periodicity indicative of active translation starting at the annotated start codon and ending at the annotated stop codon. Ribosome protected fragment length is of a consistent length with other studies (E,F). Top row is data from 4 replicates of $C$. neoformans $H 99$, bottom row form 2 replicates of $C$. deneoformans JEC21. These figures were made using RiboViz (86). 


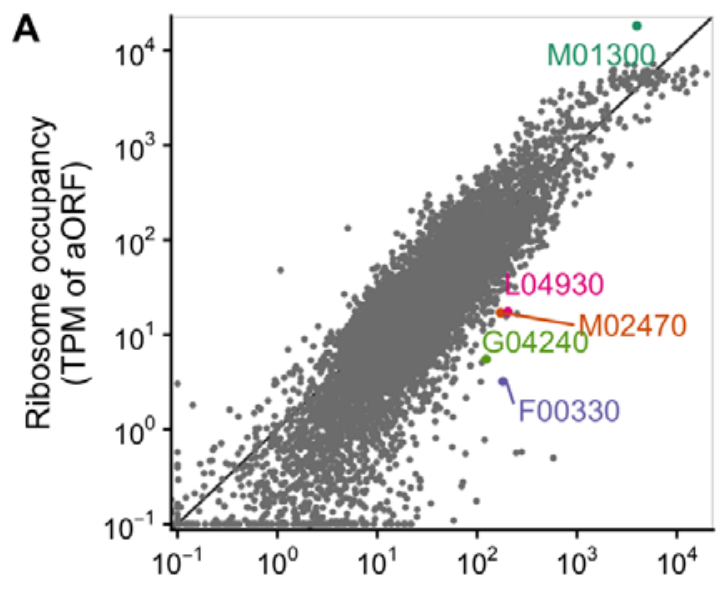

RNA abundance (TPM of aORF)

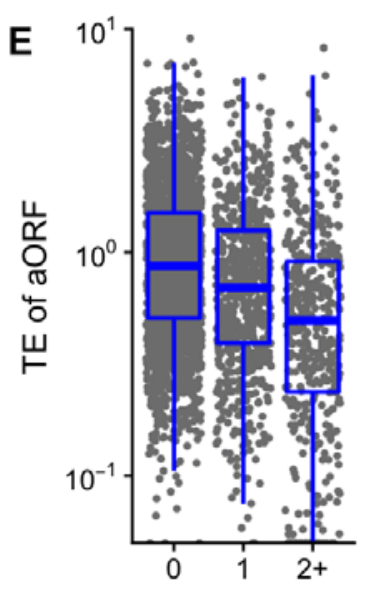

uAUG count

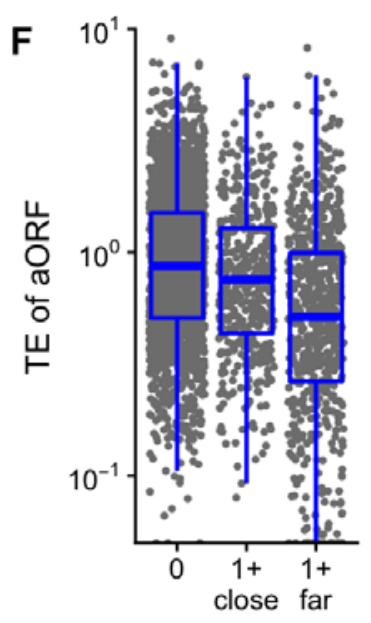

uAUG count

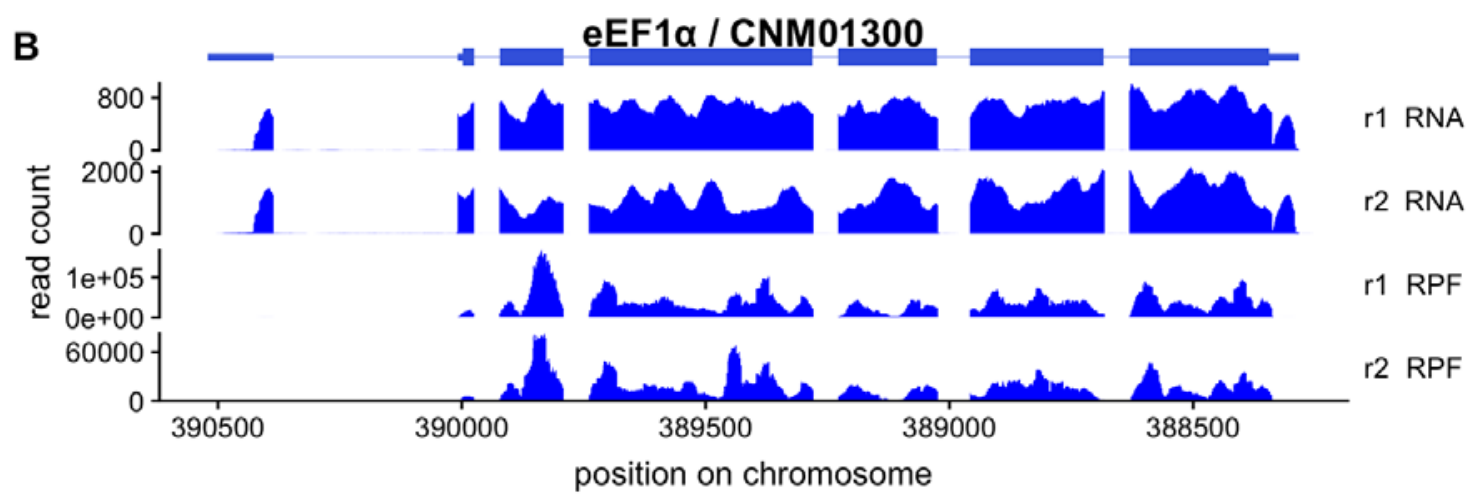

C
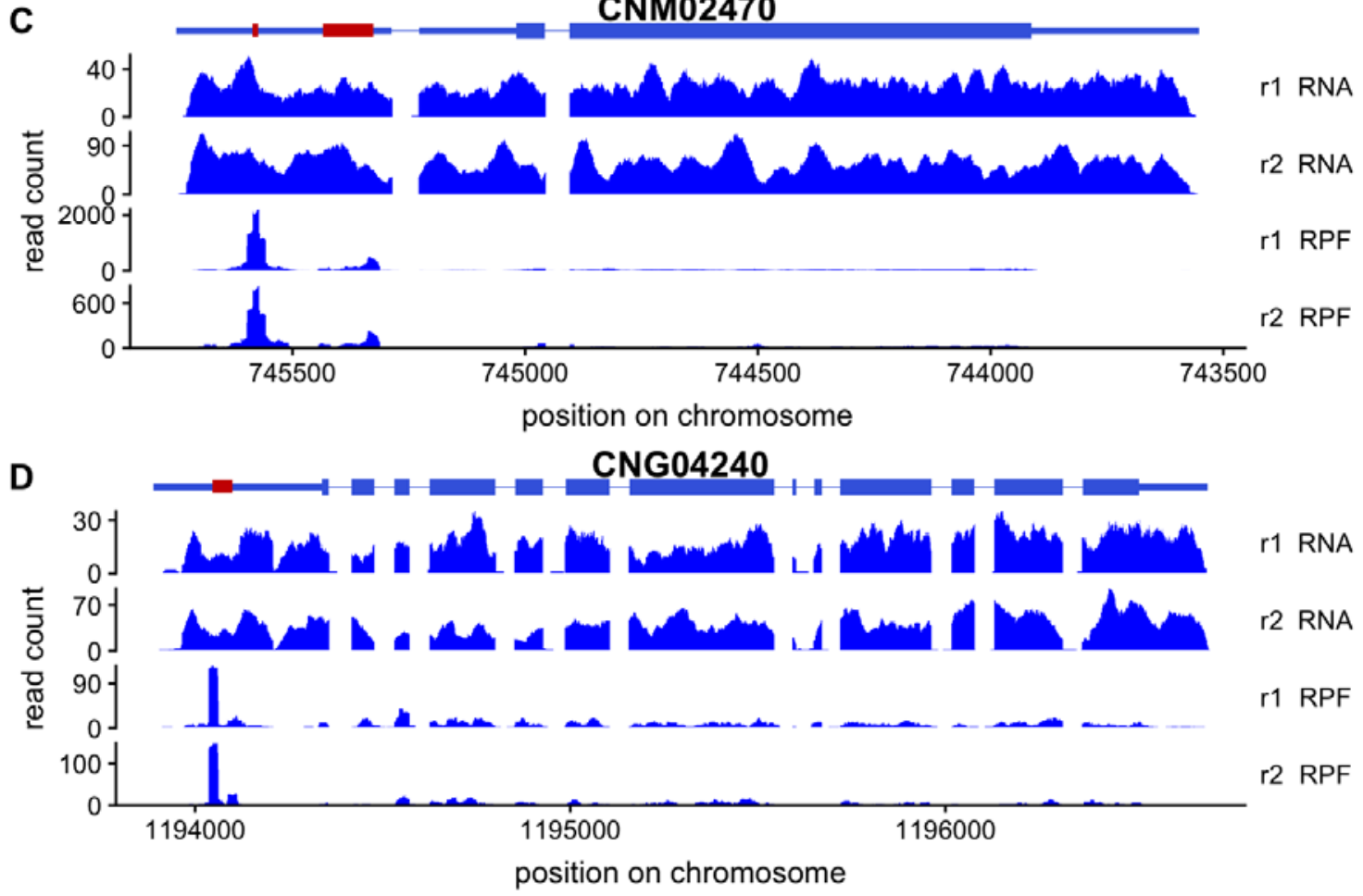
Figure S2.2, related to figure 2: Upstream AUGs repress translation in $C$. deneoformans. A, translation regulation of annotated ORFs (aORFs) in C. deneoformans JEC21 growing exponentially in YPD at $30^{\circ} \mathrm{C}$. Ribosome occupancy is plotted against the RNA abundance, both calculated in transcripts per million (TPM) on the aORF. B, UAUGs are associated with lower translation efficiency (TE) of annotated ORFs, measured as the ratio of ribosome occupancy to RNA-seq reads. C, only UAUGs far from the transcription start site are associated with low TE. A gene is in the " $1+$ far" category if it has at least one uAUG more than 20nt from the TSS, "1+ close" if all UAUGs are within 20nt of the TSS. D-F, Examples of ribosome occupancy profiles along select RNAs highlighted in A (others are shown in Fig S2B). D, Translation elongation factor eEF2/CNM01300 (CNAG_06125 homolog) has high ribosome occupancy in the annotated ORF. Translationally repressed mRNAs CNM02470 (CNAG_06246 homolog, E) and CNG04240 (CNAG_03140 homolog, F) have high ribosome occupancy in uORFs in the transcript leader (red), and low ribosome occupancy in the aORF. Only the first of 5 uORFs in CNG04240 is shown, and only transcript isoform t01 is shown, excluding the annotated TL intron in isoform t02. 

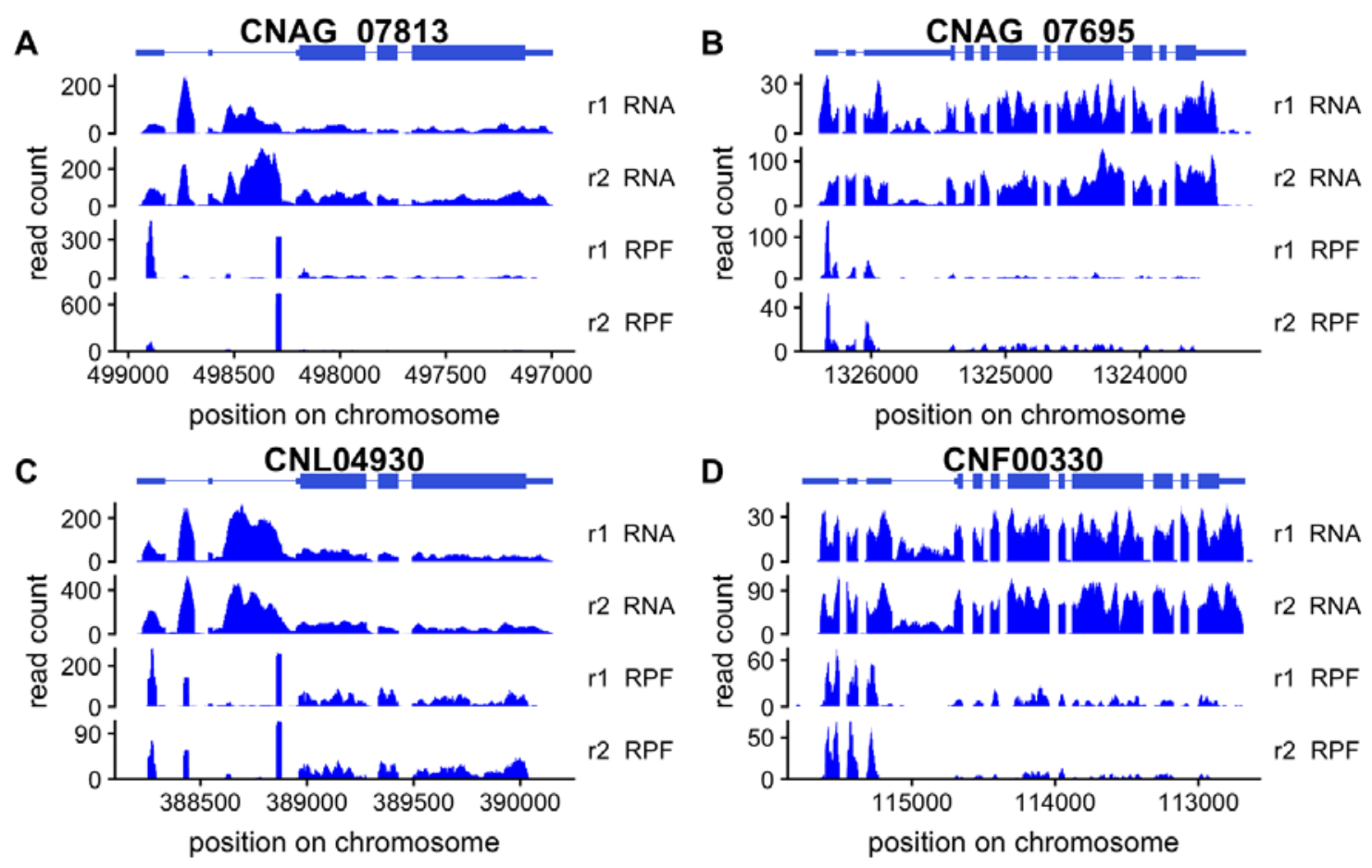

Figure S2.3, related to figure 2: Further examples of upstream AUG and 5'-end regulation in C. neoformans and C. deneoformans. CNAG_07813 (A) and CNL04930 (C) are paralogs, and in addition to an upstream ORF with ribosome occupancy, they have an intronically encoded non-coding RNA in the TL. CNAG_07695 (B) and CNF00330 (D) are paralogs, and in addition to an upstream ORF with ribosome occupancy, they have an alternatively-spliced intron in the TL that is not occupied by ribosomes. 
A
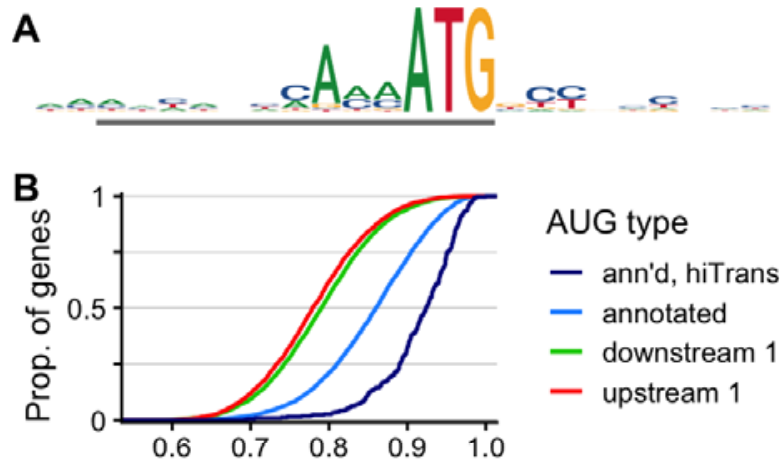

Kozak score

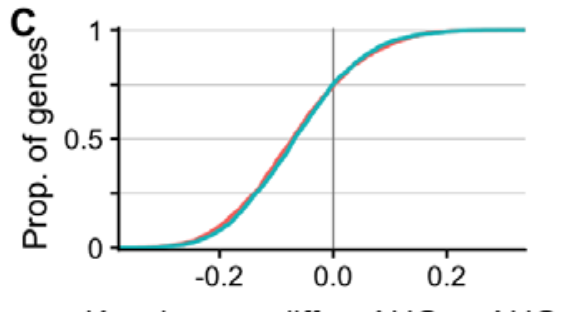

Kozak score diff. $\mathrm{u}_{1} A \cup G$ - aAUG
AUG type

- ann'd, hiTrans

- annotated

- downstream 1

- upstream 1

AUG context

- narrow (-4)

- wide $(-10)$
D

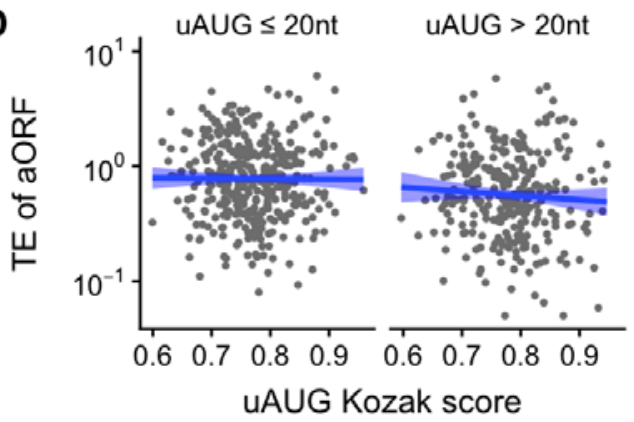

E

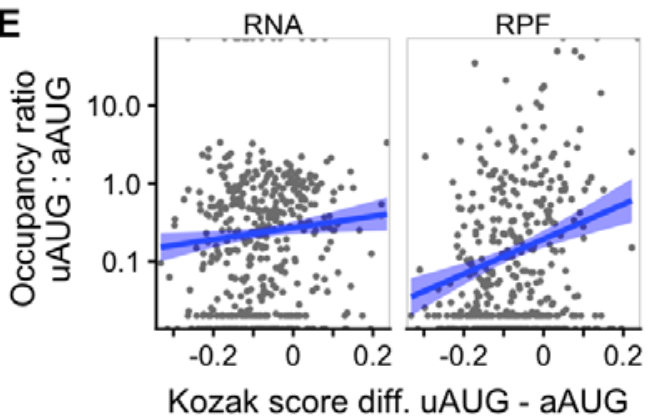

Figure S3.1, related to Figure 3: AUG sequence context is associated with translation in C. deneoformans. As for Fig 3, but with data from C. deneoformans JEC21. 


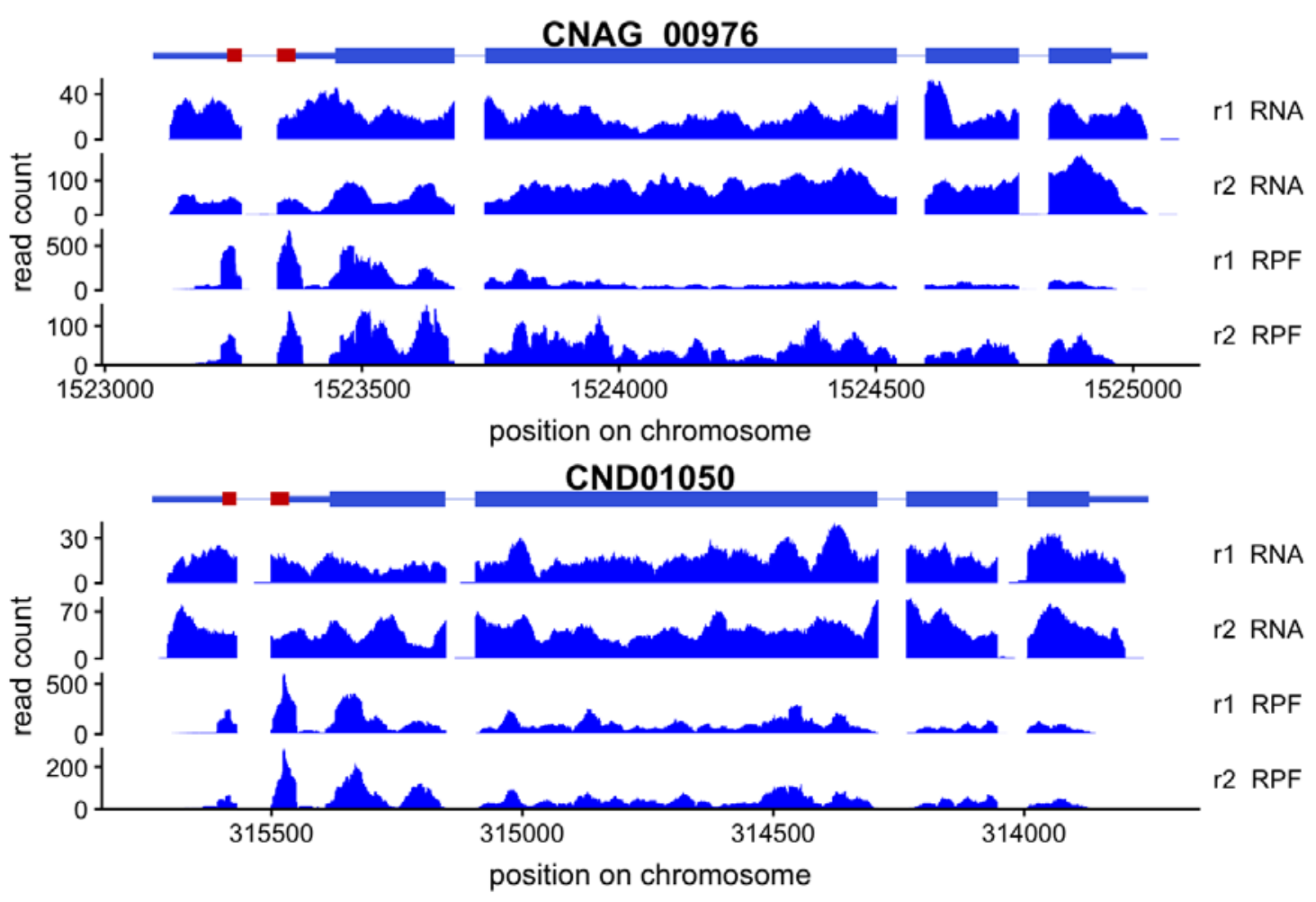

Figure S4.1, related to Figure 4: Carbamoyl-phosphate synthase CPA1 homologs have a conserved UORF that is occupied by ribosomes in $C$. neoformans (A) and $C$. deneoformans (B). 
A
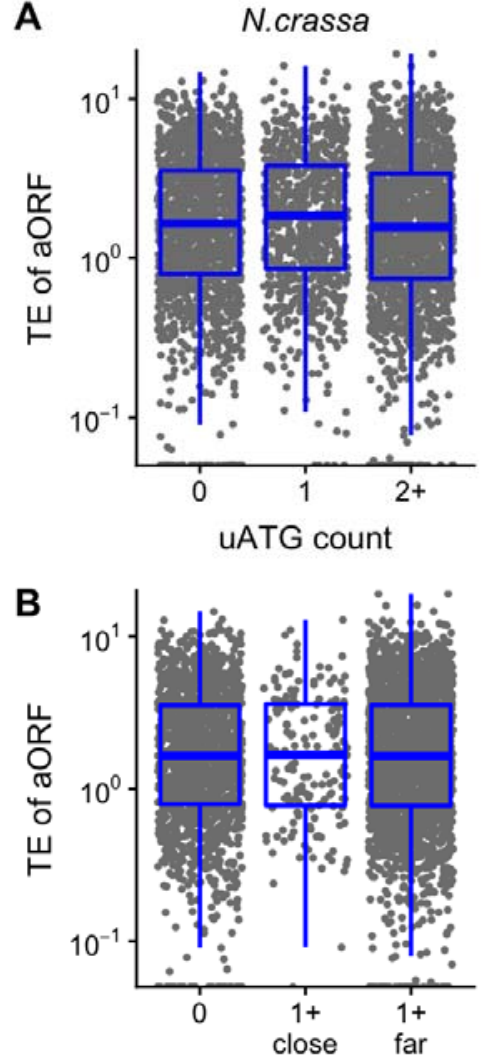

UATG count and position relative to TSS
E
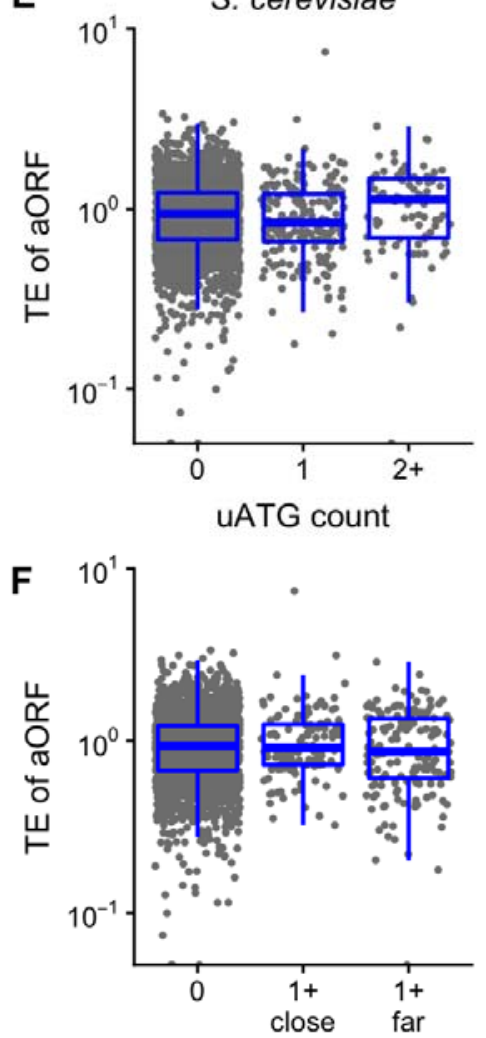

uATG count and position relative to TSS
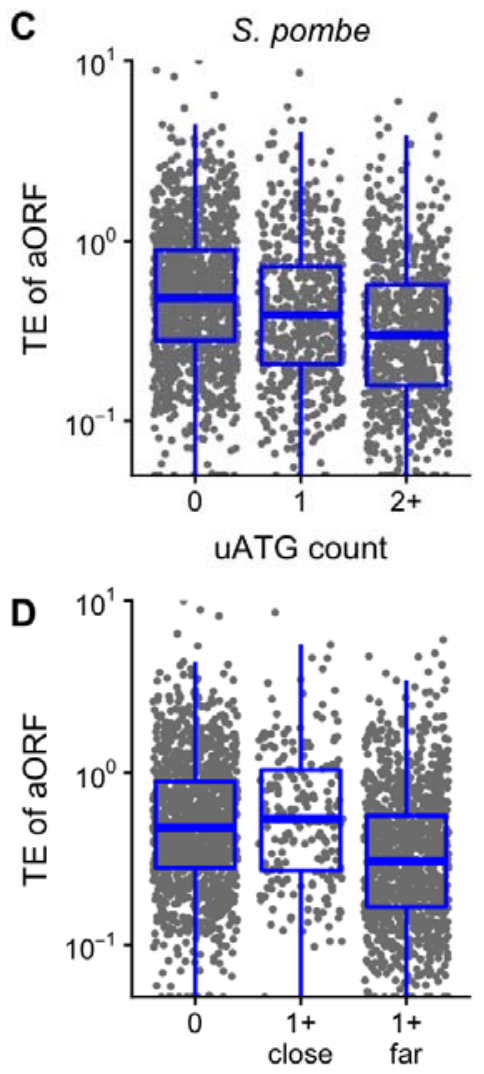

uATG count and position relative to TSS

Figure S5.1, related to figure 5: Effect of UATGs on translational efficiency in N. crassa, $S$. pombe, and S. cerevisiae. 
A

PUS1 / CNAG 06353

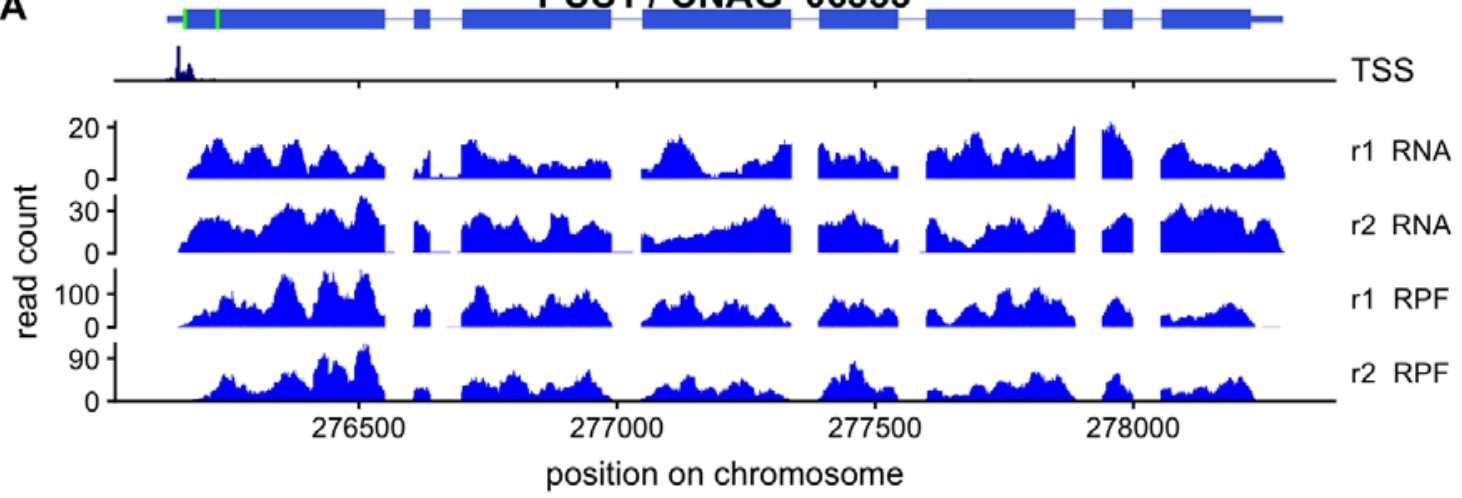

B

GLO1/CNAG 04219

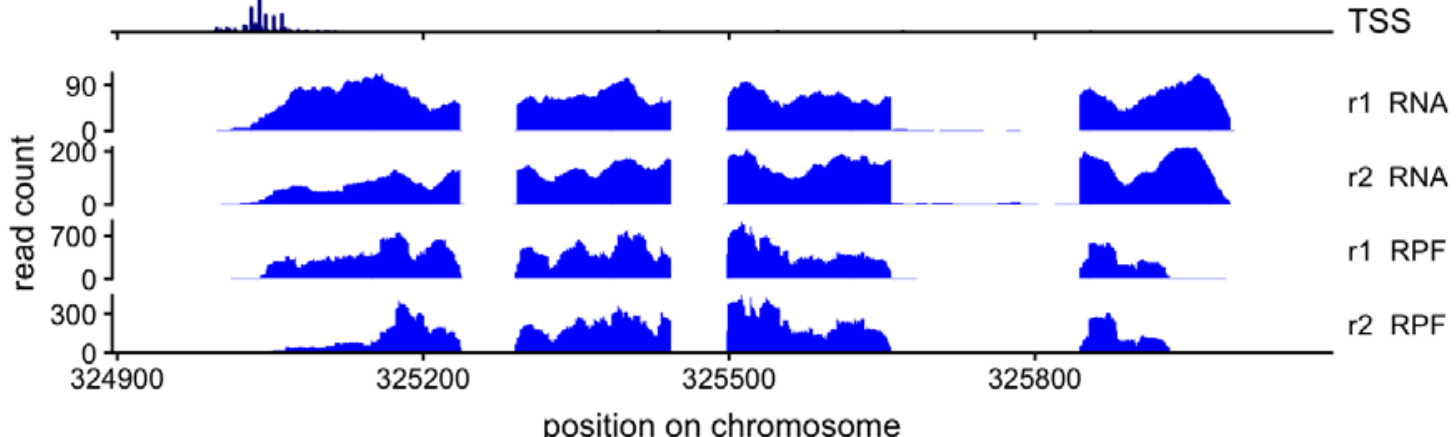

C

GLO2 / CNAG 01128

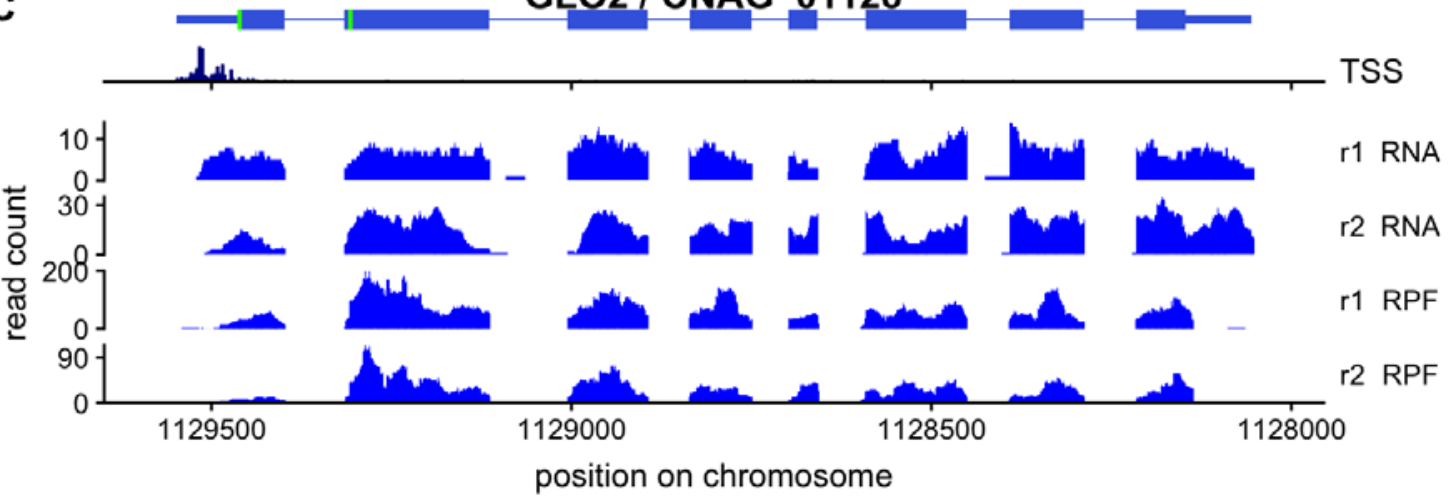

Figure S6.1, related to Figure 6: Ribosome profiles of $C$. neoformans genes with predicted dual-localization specified by alternative $\mathrm{N}$-termini. 

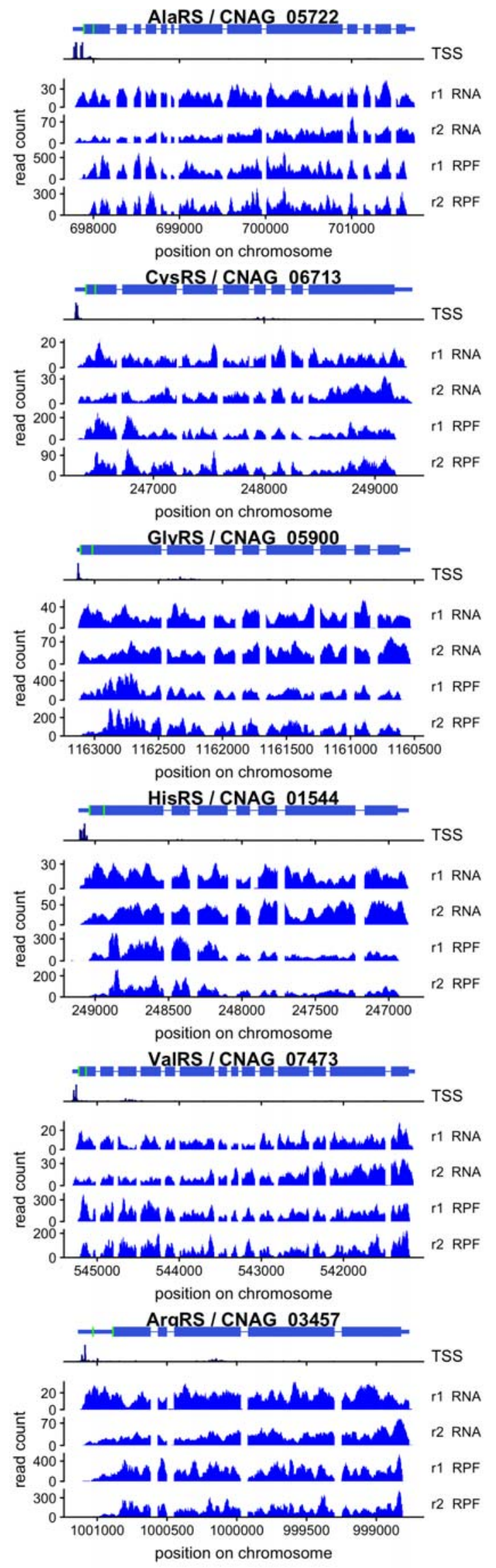
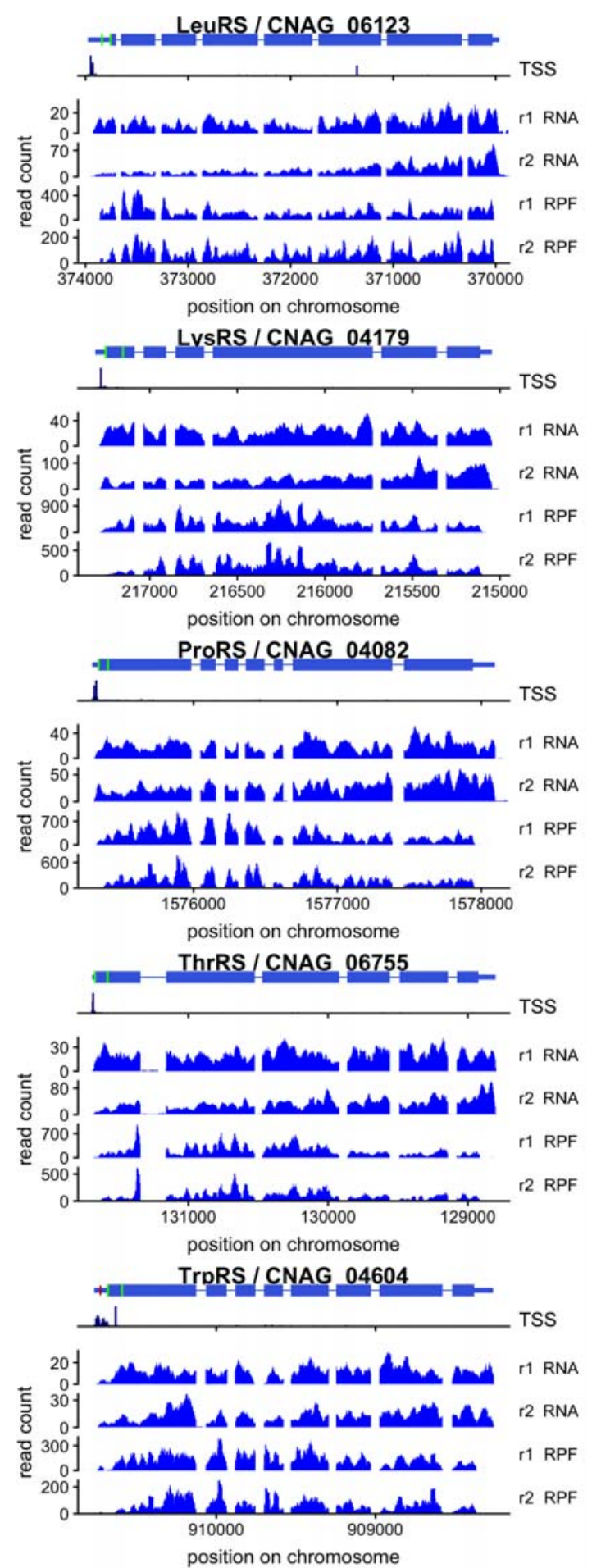
bioRxiv preprint doi: https://doi.org/10.1101/654046; this version posted July 3, 2019. The copyright holder for this preprint (which was not certified by peer review) is the author/funder, who has granted bioRxiv a license to display the preprint in perpetuity. It is made available under aCC-BY 4.0 International license.

Figure S7.1, related to Figure 7: Ribosome profiles along the 11 . neoformans aaRS genes with predicted dual-localization. Predicted start codons are shown in green, and the UORF of TrpRS in dark red. 
A

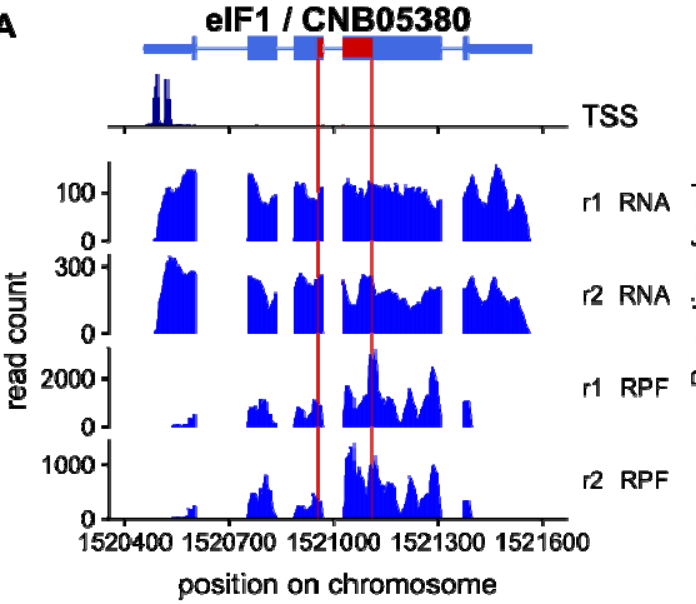

B

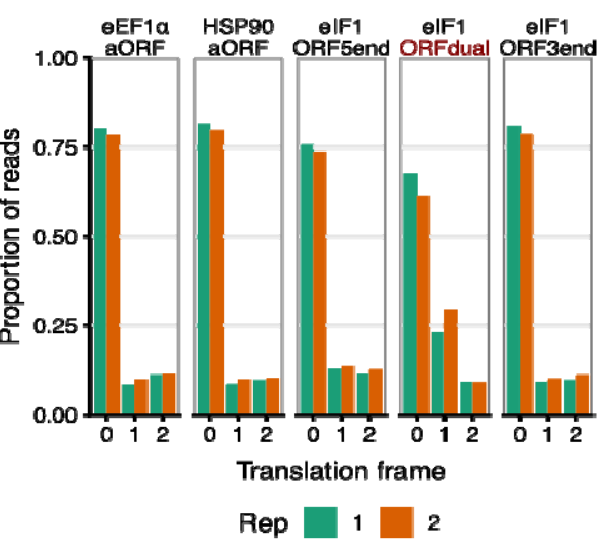

C

elF5 / CNC02150 TSS

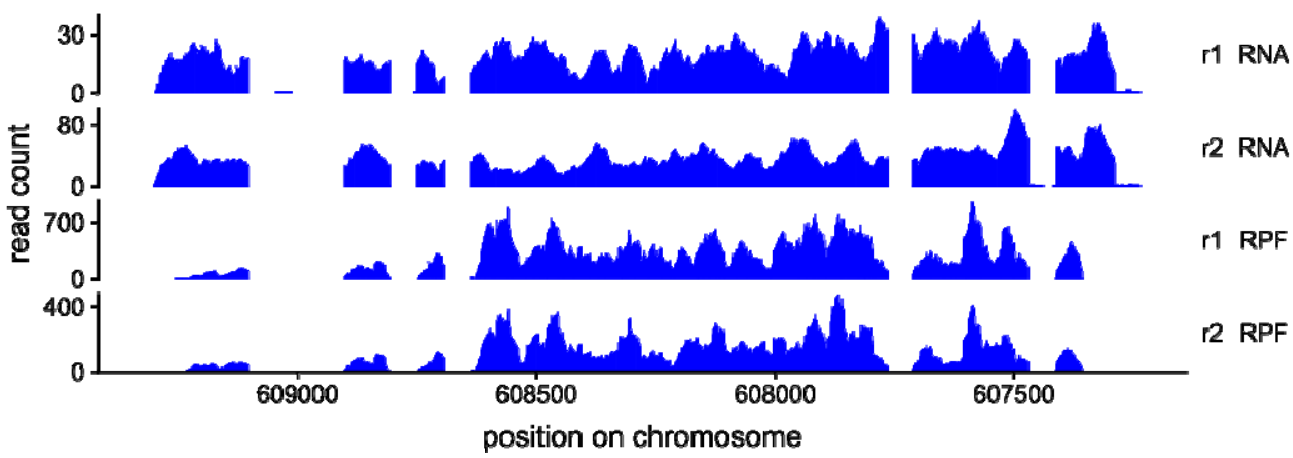

Figure S8, related to Figure 8: Translation initiation factors elF1 and elF5 are regulated by alternate start codon usage in $C$. deneoformans. See figure 8 for legend. 
bioRxiv preprint doi: https://doi.org/10.1101/654046; this version posted July 3, 2019. The copyright holder for this preprint (which was not certified by peer review) is the author/funder, who has granted bioRxiv a license to display the preprint in perpetuity. It is made available under aCC-BY 4.0 International license.

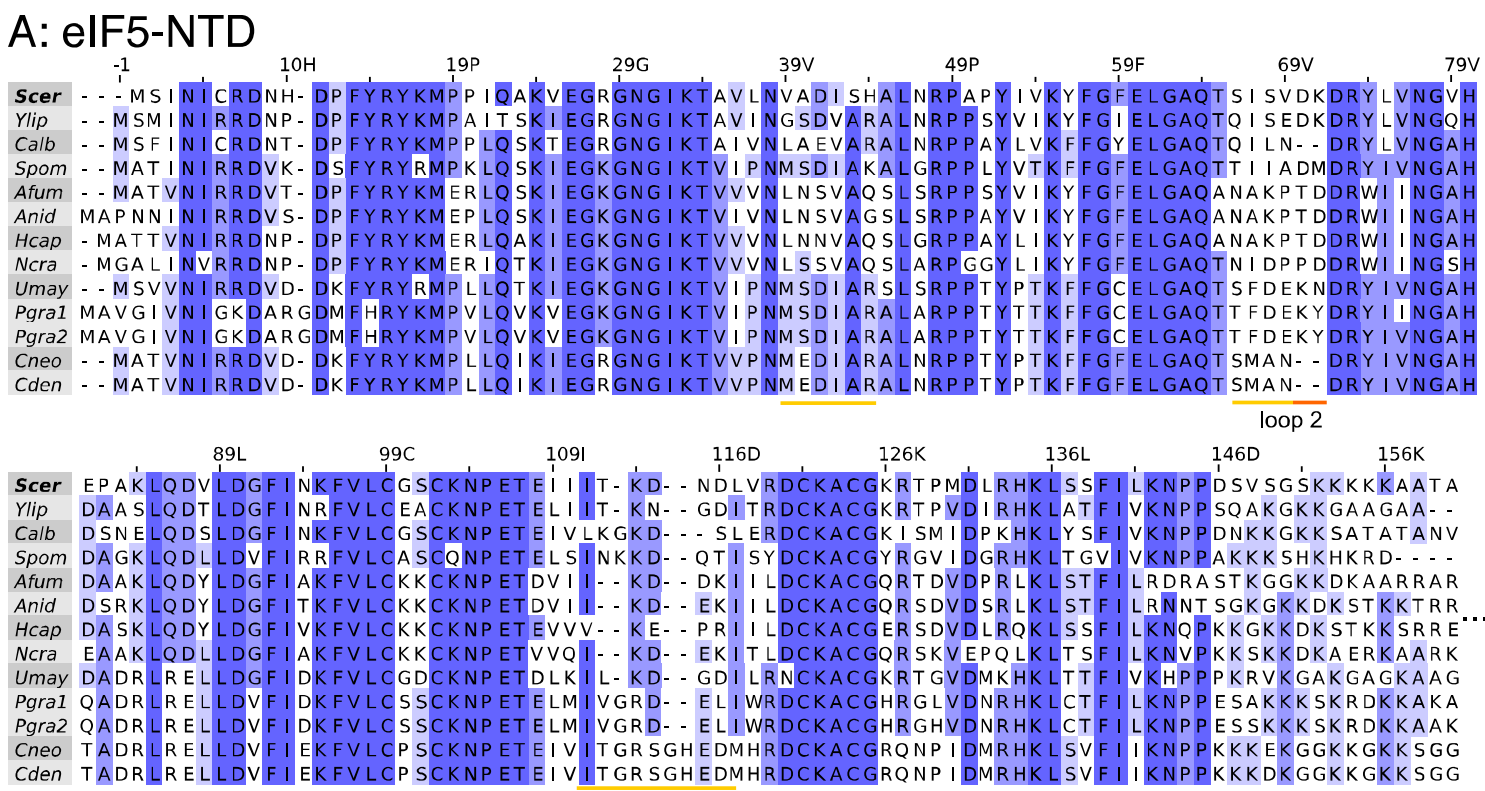

\section{B: elF1A}

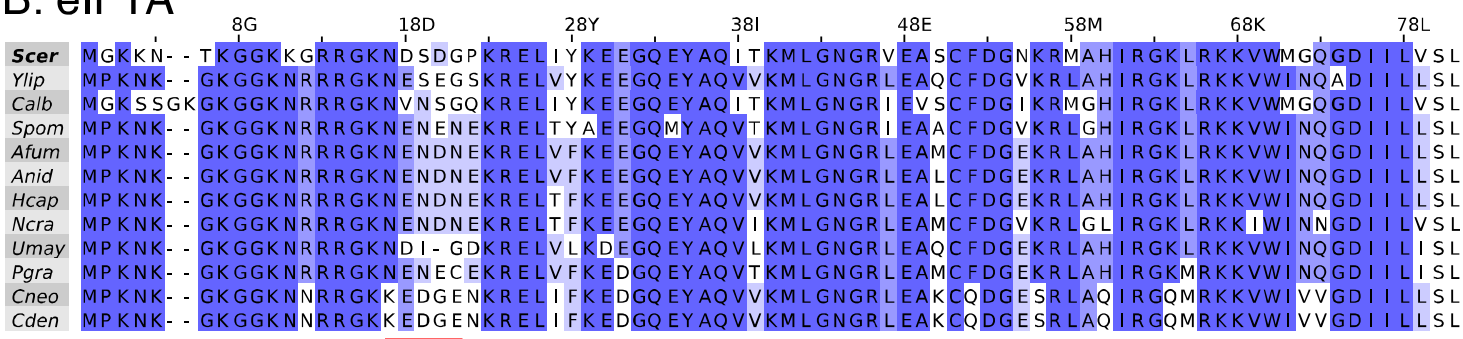

$88 \mathrm{Q}$

98D

$108 \mathrm{E}$

$118 T$

$128 \mathrm{D}$

$138 \mathrm{E}$

$147-$

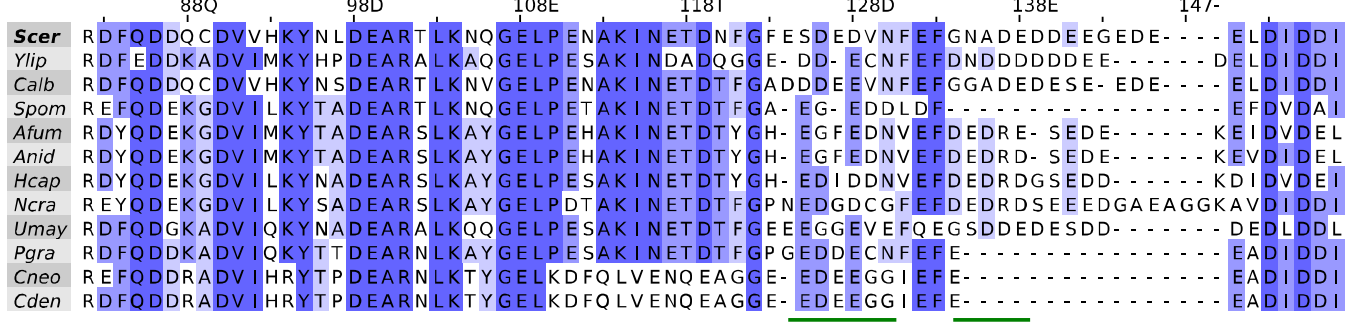

Mutations in Scer increase (Ssu-) or decrease (Sui-) fidelity of AUG selection

Figure S9.1, related to Figure 9: multiple sequence alignments of elF5-NTD and elF1A from select fungi. Sequences are numbered according to the $S$. cerevisiae homologs. 
Table S1: Sequencing and annotation numbers (Excel table).

Table S2: Differential expression results in Cryptococcus deneoformans upf1士.

Table S3: Cytoplasmic ribosomal proteins in 6 fungal species.

Table S4: Genes with score d1AUG - aAUG > 0.1, $n=167$ (Excel table).

Table S5: List of aaRS in Cryptococcus and select fungi (Excel table).

Table S6: Initiation contexts of annotated and downstream AUGs in 9 Cryptococcus aaRSs

Table S7: Initiation factor 3 components in 12 fungal species. (Excel table)

We show homologs of all human elF3 components elF3a-elF3m, assembled mostly from PANTHERdb (51). Note that the $C$. neoformans homolog PRT1 is expressed in two nonidentical paralogs at the mating-type locus, so does not have a systematic ORF name.

Table S8: rRNA subtraction oligos used in ribosome profiling. 